\title{
Dilatancy in dense suspensions of model hard-sphere-like colloids under shear and
} extensional flow

Andrade, Ricardo J. E.; Jacob, Alan R.; Galindo-Rosales, Francisco J.; Campo-Deaño, Laura; Huang, Qian; Hassager, Ole; Petekidis, George

Published in:

Journal of Rheology

Link to article, DOI:

$10.1122 / 1.5143653$

Publication date:

2020

Document Version

Publisher's PDF, also known as Version of record

Link back to DTU Orbit

Citation (APA):

Andrade, R. J. E., Jacob, A. R., Galindo-Rosales, F. J., Campo-Deaño, L., Huang, Q., Hassager, O., \& Petekidis, G. (2020). Dilatancy in dense suspensions of model hard-sphere-like colloids under shear and extensional flow. Journal of Rheology, 64(5), 1179-1196. https://doi.org/10.1122/1.5143653

\section{General rights}

Copyright and moral rights for the publications made accessible in the public portal are retained by the authors and/or other copyright owners and it is a condition of accessing publications that users recognise and abide by the legal requirements associated with these rights.

- Users may download and print one copy of any publication from the public portal for the purpose of private study or research.

- You may not further distribute the material or use it for any profit-making activity or commercial gain

- You may freely distribute the URL identifying the publication in the public portal 


\section{Dilatancy in dense suspensions of model hard-sphere-like colloids under shear and extensional flow}

Ricardo J. E. Andrade, Alan R. Jacob, Francisco J. Galindo-Rosales, Laura Campo-Deaño, Qian Huang, Ole Hassager, and George Petekidis

Citation: Journal of Rheology 64, 1179 (2020); doi: 10.1122/1.5143653

View online: https://doi.org/10.1122/1.5143653

View Table of Contents: https://sor.scitation.org/toc/jor/64/5

Published by the The Society of Rheology

\section{ARTICLES YOU MAY BE INTERESTED IN}

Analysis of linear viscoelasticity of aging soft glasses

Journal of Rheology 64, 1197 (2020); https://doi.org/10.1122/8.0000099

Yielding, thixotropy, and strain stiffening of aqueous carbon black suspensions

Journal of Rheology 64, 955 (2020); https://doi.org/10.1122/8.0000028

A constitutive model for sheared dense suspensions of rough particles

Journal of Rheology 64, 1107 (2020); https://doi.org/10.1122/8.0000039

Effects of elasticity and flow ramp up on kinetics of shear banding flow formation in wormlike micellar fluids Journal of Rheology 64, 1161 (2020); https://doi.org/10.1122/8.0000010

Interplay of edge fracture and shear banding in complex fluids

Journal of Rheology 64, 1147 (2020); https://doi.org/10.1122/8.0000086

Linear wormlike micelles behave similarly to entangled linear polymers in fast shear flows

Journal of Rheology 64, 879 (2020); https://doi.org/10.1122/8.0000003

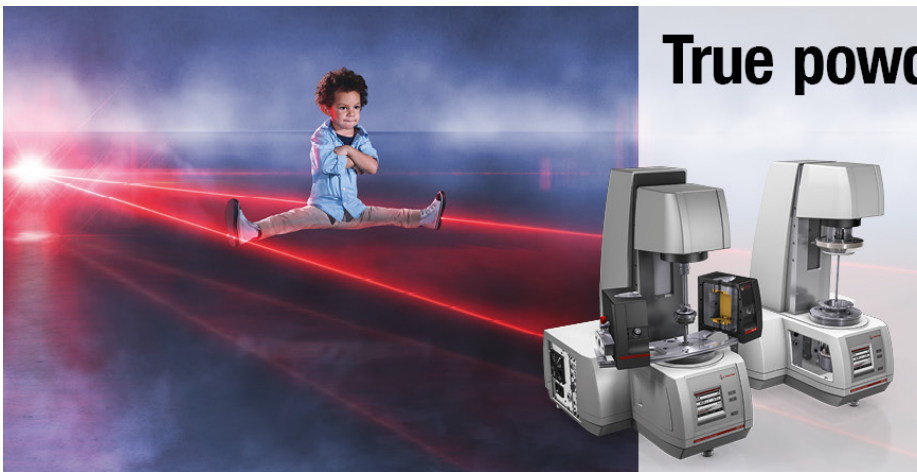




\title{
Dilatancy in dense suspensions of model hard-sphere-like colloids under shear and extensional flow
}

\author{
Ricardo J. E. Andrade, ${ }^{1,2, a)}$ Alan R. Jacob, ${ }^{2}$ Francisco J. Galindo-Rosales, ${ }^{3}$ Laura Campo-Deaño, ${ }^{3}$ \\ Qian Huang, ${ }^{4}$ Ole Hassager, ${ }^{4}$ and George Petekidis ${ }^{2}$ \\ ${ }^{1}$ MackGraphe-Graphene and Nanomaterials Research Center, Mackenzie Presbyterian University, São Paulo, Brazil \\ ${ }^{2}$ Institute of Electronic Structure and Laser (IESL), Foundation for Research and Technology-Hellas (FORTH), \\ Heraklion, Crete, Greece \\ ${ }^{3}$ Centro de Estudos de Fenómenos de Transporte (CEFT), Faculdade de Engenharia da Universidade do Porto, Porto, \\ Portugal \\ ${ }^{4}$ Department of Chemical and Biochemical Engineering, Technical University of Denmark, Lyngby, Denmark
}

(Received 26 December 2019; final revision received 4 August 2020; published 1 September 2020)

\begin{abstract}
Dense suspensions of model hard-sphere (HS)-like colloids, with different particle sizes, are examined experimentally near and in the glass state, under shear and extensional rheology. Under steady shear flow, we detect both continuous and discontinuous shear thickening (DST) above a critical shear rate (or shear stress), depending on the particle size and volume fraction. Start-up shear experiments show stress overshoots in the vicinity of the onset of DST indicative of microscopic structural changes, while the sample macroscopically shows dilatancy effects. Measurement of shear and normal stresses together with direct video imaging of the sample probes the appearance of the positive first normal stress difference, $N_{1}$, at the onset of shear thickening at high shear rates and glassy states. This is followed by dilatancy effects accompanied by large fluctuations of shear and normal stress and stick-slip phenomena. Similarly, under extensional flow probed by capillary breakup and filament stretching setups, we find liquidlike response for low strain rates, while above a critical strain rate, the samples exhibit a solidlike behavior where thickening is accompanied by a macroscopic dilatancy and granulation. Monitoring the filament thinning processes under different conditions (volume fractions and strain rates), we have created a state diagram where all responses of a HS suspension (liquidlike, shear thinning, shear thickening, and dilatant) are shown. We, finally, compare the shear thickening response of these HS-like suspensions and glasses in shear with that in the extensional flow. (C) 2020 The Society of Rheology. https://doi.org/10.1122/1.5143653
\end{abstract}

\section{INTRODUCTION}

Concentrated colloidal suspensions often show an increase in the shear viscosity, beyond a critical shear rate, a phenomenon that is known as shear-thickening. Such a behavior has been observed in a wide variety of suspensions [1,2] and can often cause damage in industrial processes by breaking pumps and damaging valuable processing equipment. Understanding and controlling shear-thickening behavior is important, not only in the design of industrial processes, but also on a wide range of innovative applications such as bullet proof body armor [3], impact protective sandwich structures [4], damping devices [5,6], and driving fluids for enhanced oil recovery [7]. Despite the importance of such a phenomenon, the mechanism behind the shear thickening of concentrated colloidal suspensions still under debate.

Different mechanisms have been proposed to explain aspects of shear-thickening over the years. The first attempt to understand the relevant microstructural rearrangements came from Hoffman [8-10], who observed that polymer particles formed layers and moved across each other at low shear rates, causing shear thinning, whereas at higher shear

\footnotetext{
a) Author to whom correspondence should be addressed; electronic mail: ricardo.andrade@mackenzie.br
}

rates, a disordered regime was related with shear-thickening. However, later, it was shown that shear-thickening can occur without necessarily an order-disorder transition taking place $[11,12]$ suggesting that such microstructural reorganization is not a prerequisite.

Brady and Bossis [13] introduced the hydrocluster mechanism. They suggested the formation of hydroclusters, temporary stress bearing aggregates of particles, that form as a result of short-range hydrodynamic forces and overcome the repulsive forces between particles during shear. Experimentally, this mechanism has been probed by rheo-optical measurements [14-16], neutron scattering [17-20], and by confocal microscopy [21]. This mechanism is being demonstrated to be responsible for the continuous shear thickening (CST), where viscosity increases gradually [13,22,23], in Brownian suspensions of moderate volume fractions [21,24]. However, this mechanism alone does not seem to predict quantitatively the discontinuous shear thickening (DST) observed experimentally, which is related to the abrupt, step rise of viscosity with the increasing shear rate.

More recently, frictional contacts have been introduced as a key underlying mechanism in DST of noncolloidal suspensions [25-28] that is expressed in extreme cases by dilation [29-32]. These studies suggest that when the applied shear stress overcomes the lubrication film, a contact between particles takes place enabling local friction between particle 
surfaces to develop. These frictional contacts produce shear stress proportional to normal stress, leading to a dramatic increase in the measured shear stress, which coincides with shear-thickening. Moreover, at sufficiently high volume fractions, particle crowding and jamming [33-36] promote frictional contacts and then dilation forces that push against boundaries, usually at a liquid-air interface, are introduced. This mechanism was able to predict the DST; however, most of the experimental data have been performed on non-Brownian suspensions.

Theoretical models also suggest that while DST in jammed states results in flow cessation, for concentrated suspension below the glass transition, DST is related with shear rate hysteresis, where a shear thickened system still flows under shear [37]. A recent experiment focused on separating the contributions from hydrodynamic interactions and contact forces using shear-reversal techniques [38] and compared viscosity behavior to friction-based models [39], or used topographically rough particles to evaluate friction effects on shear-thickening [40]. The signature difference between friction and lubrication forces is the stress anisotropy originating from the shearinduced microstructure involved in these two types of mechanisms [40,41]. This anisotropy is reflected in the first normal stress difference $N_{1}$ as it was shown by simulations based on hydrodynamics interactions showing shear-induced distortions and short ranged lubrications forces lead to $N_{1}<0$ $[22,24,42,43]$. It was also demonstrated that the sign of $N_{1}$ does not change with repulsive interactions or elastic deformations $[36,41,44]$. On the other hand, positive normal stress have been related to dilatancy, as a feature of dense and frictional granular materials $[31,45]$. It is important to refer that, in these works, dilatancy is a direct physical consequence of an outward-pushing force (positive normal stress), rather than first normal stress difference $N_{1}[46,47]$. At high volume fractions, contact forces lead to dilation $\left(N_{1}>0\right)$, due to the anisotropy of the force chain network [48], as it occurs in granular systems [49]. Due to the experimental difficulty, studies providing accurate $N_{1}$ values in shear thickening suspensions are limited, with most experiments showing $N_{1}<0$ [50-53], with the exception of experiments with rough particles [33]. Recently, it was demonstrated that, at moderate volume fractions, negative contributions of $N_{1}$ from lubrication forces can hide positive frictional contributions, but at significant high volume fractions and stresses, frictional interactions become dominant causing positive $N_{1}$ [41,54].

As stated above, measuring the first normal stress difference can provide valuable information of the micromacrorelationship; moreover, by subjecting a shearthickening suspension to varying types of flow deformations, it is possible to access the dynamics and physics of the suspension. In this context, extensional flows are also very important to colloidal suspensions. Although concentrated colloidal suspensions have attracted considerable attention in shear flows, the studies in extensional flows are still limited.

An extensional flow usually enhances the nonlinear behavior and exhibits dynamics and structural changes different from the shear flow [47]. Extensional flows are of great interest because many industrially relevant processes, such as agro-chemical spraying, enhanced oil recovery, coating flows, fiber spinning, and food processing, are dominated by the fluids' extensional properties [7,55,56]. White et al. [57] investigated the extensional rheology of corn starch in water suspensions using a home-built filament stretching extensional rheometer (FiSER) and capillary breakup extensional rheometer (CaBER), to study the mechanism of strain hardening. A strong extensional hardening behavior, which means an increase in the extensional viscosity with strain rate, was observed beyond a critical extension rate, which was related to the aggregation of particle clusters. Chellamuth et al. [58] studied the extensional rheology of dispersion of fumed silica particles suspended in polypropylene glycol with low molecular weight using a FiSER setup combined with light scattering measurements to elucidate the microstructure evolution during flow. Beyond a critical extensional rate, a dramatic increase in the strain-hardening effect was observed, similar to the shear thickening transition observed under shear, however with a higher magnitude and at reduced critical deformation rates. Light scattering measurements showed that the extensional hardening was due to the alignment of nanoparticles and the formation of large aggregates in the flow direction. These were the first direct observations of hydrodynamically induced clustering in the extensional flow. Smith et al. [59] analyzed the flow behavior of concentrated suspensions of nearly hard-sphere (HS) colloids under extensional flow with a single particle size at different volume fractions. They showed that, above a critical extension rate, the suspension jams and dilates, by expanding its volume, exhibiting dramatic granulation as demonstrated by a macroscopic lumpiness and rough surface appearance, followed by a solidlike break up behavior. Besides the above studies until now, there is a lack of information that relates the particle size in concentrated colloidal HS suspensions with the strain hardening and consequent dilatancy behavior in extensional flow, and its direct comparison with thickening under shear flow.

In this work, we present a comparison of the rheological behavior of concentrated colloidal dispersions under shear and extensional flow, using model nearly HS particles of different sizes and volume fractions in the concentrated and glassy states. To understand the underlying mechanism governing the flow response of these suspensions, a complete rheological characterization is necessary together with an investigation of the relation of their microscale and macroscale behavior under different flow conditions. Toward this goal, we study the rheological response of model nearly HS colloids of three different sizes all at the colloidal scale (i.e., where Brownian motion is dominant at rest), under shear and extensional flow. Depending on the particle size and rate of deformation, different behaviors were observed. The combination of the information provided by both types of flow allows a comprehensive characterization of the system, providing insights into the mechanism related with shearthickening. Our work here complements recent studies on shear thickening by addressing how ideas of dilatancy, which were mostly developed for noncolloidal particles, apply to colloidal suspensions, and presents an example where an interplay between hydrodynamic lubrication related negative $N_{1}$ and presumably friction induced positive $N_{1}$ and dilatancy effects takes place in shear experiments. We further relate 
such a shear thickening response with its corresponding findings in extensional rheometry.

\section{EXPERIMENTAL SECTION}

\section{A. Samples}

Colloidal glasses containing nearly HS particles with sizes approximately $R \approx 689,405$, and $137 \mathrm{~nm}$ and polydispersity ranging from $7 \%$ to $10 \%$, were prepared. Particles are made of polymethyl methacrylate (PMMA) sterically stabilized by a thin grafted layer $(\approx 10 \mathrm{~nm})$ of poly-12-hydroxystearic acid (PHSA) chains inducing steric repulsion that stabilizes them against van der Waals attractions creating an almost ideal HS interaction. PMMA particles (density $\approx 1180 \mathrm{~kg} / \mathrm{m}^{3}$ ) were dispersed in squalene (viscosity $=0.015 \mathrm{~Pa} \mathrm{~s}$ ), a nonvolatile solvent with a refractive index of 1.494, very similar PMMA, therefore minimizing any residual van der Waals attractions [60]. The surface tension and density of squalene are $29.2 \mathrm{mN} / \mathrm{m}$ and $848 \mathrm{~kg} / \mathrm{m}^{3}$ at $25^{\circ} \mathrm{C}$, respectively. The largest particles $(R \approx 689 \mathrm{~nm})$ were used in the glassy $(\varphi \approx 0.60)$ and in the supercooled regime $(\varphi \approx 0.56)$, as well as the particles of $R \approx 137 \mathrm{~nm}$, while the particles of $R \approx 405 \mathrm{~nm}$ were used only for measurements in the glassy regime $(\varphi \approx 0.60)$. These volume fractions were chosen with the aim to investigate the shear thickening behavior in the glassy regime, where the onset is detected at lower stresses and shear rates $[2,16,61]$. The bare Brownian time defined as $t_{B}=$ $6 \pi \eta_{S} R^{3} / k_{B} T$ was used in the nondimensional shear rate, $P e=$ $\dot{\gamma} t_{B}$ that quantifies the interplay between Brownian motion and shear, and similarly in the nondimensional extension rate, $P e_{e x t}=\dot{\varepsilon} t_{B}$. The Brownian times for the particles with radii $689 \mathrm{~nm}, 405 \mathrm{~nm}$, and $137 \mathrm{~nm}$ are $22.87 \mathrm{~s}, 4.645 \mathrm{~s}$, and $0.0179 \mathrm{~s}$, respectively.

\section{B. Shear rheology}

A cone and plate geometry attached to an Anton Paar MCR 501 rheometer was used for the shear experiments. More specifically, we used a glass cone with a diameter $2 r=40 \mathrm{~mm}$, angle $=1.967^{\circ}$, and truncation gap $=176 \mu \mathrm{m}$. The temperature was fixed at $20^{\circ} \mathrm{C}$ with a Peltier based thermostat. Dynamic frequency sweep experiments together with flow curves provide a complete characterization of the rheological behavior of the samples. Start-up shear measurements at a fixed shear rate were performed to follow the transient response. All tests were conducted after shear rejuvenation at $\dot{\gamma}=0.5 \mathrm{~s}^{-1}$ for $300 \mathrm{~s}$ followed by a waiting time of $200 \mathrm{~s}$. In addition, a high-speed Basler camera was used to visualize the edge effects during shear experiments to relate shear thickening with dilatancy and edge fracture effects. Images were recorded every $100 \mathrm{~ms}$ for a specific amount time depending on the duration of the shear experiment.

The first normal stress difference $N_{1}=\sigma_{x x}-\sigma_{y y}$, where $\sigma_{x x}$ is the stress acting normal to the shear plane and $\sigma_{y y}$ is the stress acting normal to the shear gradient plane, extracted from the axial force measured by the transducer, $2 F_{y}$ $=N_{1} \pi r^{2}$ [46,47]. However, the above holds under certain conditions including that, in spherical coordinates, the velocities in the radial and $\theta$ directions are zero (in addition to the cone angle being small and the liquid boundary spherical). Hence, while these conditions can be assumed true in the shear thinning regime, they break down when the sample dilates. Thus, to avoid any confusion, we clarify that $N_{1}$ documented here can be considered as the first normal stress difference only up to the point where these conditions hold, i.e., before dilatancy sets in, while when dilatancy is present, it represents the normal (axial) force, $F_{N}$, per unit area or normal stress, $\sigma_{N}$. The reported values of the shear and normal stress were determined during transient measurements, in conjunction with direct imaging of the sample, at a time before dilatation and edge instabilities set-in (see Figs. 6 and 7).

\section{Extensional rheology}

The rheological characterization of these concentrated colloidal suspensions under extensional flow conditions was performed at $23{ }^{\circ} \mathrm{C}$ by means of both extensional rheometers available in the market for characterizing the extensional properties of complex liquids [56], i.e., the capillary breakup extensional rheometer (Haake CaBER-1) and the filament stretching rheometer (FSR) (VADER 1000 by Rheofilament).

In the CaBER experiments, an extensional step-strain is imposed, and the filament, subsequently, thins exclusively under the influence of capillary forces. The extensional strain rate is the result of a balance between the interfacial tension and extensional properties of the liquid. In the CaBER device, a laser micrometer uniquely measures the time evolution of the diameter of the thinning filament at the midplane, $D_{\text {mid }}(t)$, which ideally coincides with the minimum diameter, $D_{\min }(t)$, and from which the strain rate at a specific time, $\dot{\varepsilon}=(d \varepsilon / d t)=-\left[\left(2 / D_{\min }(t)\right)\left(d D_{\min }(t) / d t\right)\right]$, and the apparent extensional viscosity, $\eta_{\text {Eapp }}=-\left[\sigma /\left(d D_{\min }(t)\right) / d t\right]$, can be calculated from [62], using the surface tension, $\sigma$. However, as it has been already reported before [63-66] that the shape of the filament depends on the interplay between the rheological response of the complex fluids and the test conditions. Therefore, the minimum diameter can be out of the midplane. It is then required to use a high-speed camera to monitor the filament thinning dynamics after a rapid extensional deformation. A digital post-processing image analysis in MATLAB allows in this way to determine $D_{\min }(t)$. The experimental setup was similar to the one in Campo-Deaño and Clasen [64], in the sense that the filament thinning processes were recorded by means of a high-speed video camera (Photron FASTCAM Mini UX100) recording up to $5000 \mathrm{fps}$ $(1280 \times 1024$ square pixel $)$ with an average image resolution of $\approx 5 \mu \mathrm{m}$ per pixel, in substitution of the laser detector. It is important to highlight that no SRM (slow retraction method) was imposed in the CaBER experiments. The configuration used in our work, however, did not allow recording the filament dynamics simultaneously with the laser-based measurement. Thus, we performed independent experiments determining $D_{\text {mid }}$ both with the laser and with camera assisted configurations. In Fig. 1(a), we show that the midplane diameter and the minimum diameter do not actually coincide when the sample thickens under the extensional flow, leading to inaccurate measurements when the determination of $D_{\text {mid }}$ is performed just with the laser assisted setup. 

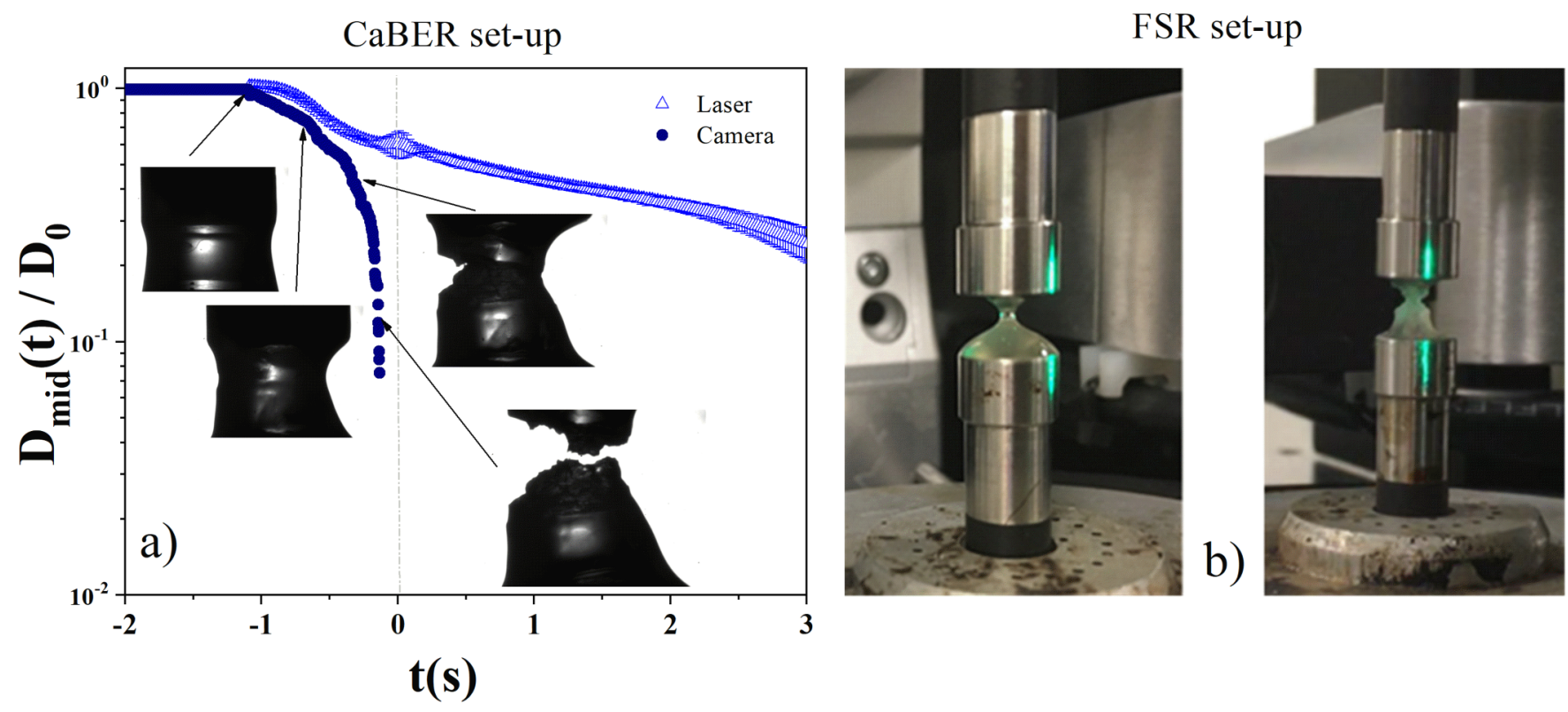

FIG. 1. (a) Time evolution of the stretched filament diameter in a CaBER experiment. Comparison between $D_{\text {mid }}(t)$ obtained with laser (light blue symbols) and $D_{\min }(t)$ obtained from the analysis of the images recorded with a high-speed camera (dark blue symbols) as a function of time during a capillary breakup extensional rheology measurement (sample with HS particles of $R \approx 405 \mathrm{~nm}$ at strain rate $\dot{\varepsilon}_{0}=1 \mathrm{~s}^{-1}$ ). (b) Images from FiSER setup with a HS glass of particles with $R \approx 689 \mathrm{~nm}$ during stretching at a low strain rate, $0.1 \mathrm{~s}^{-1}$, showing a liquidlike behavior (left) and a high strain rate, $1.0 \mathrm{~s}^{-1}$, showing solidlike behavior (right).

For this reason, hereafter only $D_{\min }$ measured with the camera will be presented in the following figures.

It is known that low values for the initial aspect ratio ( $\Lambda_{0}=2 h_{0} / D_{0}$ ), where $h_{0}$ is the initial distance between the end-plates and $D_{0}$ is the initial sample diameter, promote inhomogeneity during the extensional flow, as the radial velocity fluctuations dominate fluid motion in the filament generating shear flow near the endplates. During the early stages of the experiment, this is proportional to $\dot{\varepsilon} / \Lambda_{0}^{2}[57,67]$. Nevertheless, due to the low surface tension of the sample, we were forced to impose $\Lambda_{0}=0.75$, since the initial gap must be smaller than the capillary length $\left(h_{0} \leq \sqrt{\sigma / \rho g}\right)$, where $\sigma$ is the surface tension, $\rho$ is the density, and $g$ is the gravitational acceleration, in order to hold the sample between the plates and run the experiments [63].

Thus, the time evolution of $D_{\text {min }}$ obtained from the CaBER has two zones. The first one, with negative times (from $-t_{s}$ to 0 , where $t_{s}$ is the streak time), corresponding to the stretching zone, in which the top endplate has to reach to the final height $\left(h_{f}\right)$ and during which the fluid flow is not purely elongational, but with an important shear contribution. The strain rate imposed to the sample is, therefore, defined as $\dot{\varepsilon}_{0}=\left(h_{f}-h_{0}\right) / h_{0} t_{s}$ (see Table I). The second zone, at positive times $(t>0)$, is where the capillary forces thinning the filament are counterbalanced by the microstructural forces of the sample until it breaks up. In this study, the total time to break the filament $\left(t_{b r}\right)$ is considered from the moment in which the top plate starts to move until the sample is not bridging the plates anymore.

The FSR imposes a velocity on the upper plate in order to induce the uniaxial extensional flow [Fig. 1(b)]. The temporal evolution of the tensile force, $F(t)$, exerted by the fluid column on the bottom plate and on the filament radius at the axial midplane, $D_{\text {min }}(t)$, are measured by a load cell and a laser based micrometer, respectively, and are used to compute the transient extensional viscosity [68].

The time evolution of the uniaxial extensional stress was measured in the FSR for each sample. The samples were loaded between the two plates with a diameter of $9 \mathrm{~mm}$. The height of the samples was around $1.75 \mathrm{~mm}\left(\Lambda_{0}=0.389\right)$. This initial aspect ratio introduces an important shear component in the flow at early stages of the stretching, as it was noted before. Unlike the CaBER, the FSR allows us to perform a two-step stretching protocol. According to White et al. [57], this protocol is similar to applying a preshear step in shear experiments on a rotational rheometer in order to erase preshear history (rejuvenation) of the sample. Here, an initial stretch at extension rate well below the onset of extensional thickening is applied, followed by a second stretch much faster until the filament breaks up. The first step thus aims to minimize the shear effects on the second step, which is the actual experimental measurement.

TABLE I. Experimental conditions imposed in the linear stretching profile imposed in the CaBER experiments.

\begin{tabular}{lccc}
\hline \hline$h_{0}(\mathrm{~mm})$ & $h_{f}(\mathrm{~mm})$ & $t_{s}(\mathrm{~s})$ & $\dot{\varepsilon}_{0}\left(\mathrm{~s}^{-1}\right)$ \\
\hline 1.5 & 4.450 & 19.66 & 0.10 \\
& & 7.866 & 0.25 \\
& 3.933 & 0.50 \\
& 1.966 & 1.00 \\
& 0.988 & 1.99 \\
& 0.655 & 3.00 \\
& 0.393 & 5.00 \\
& 0.039 & 50.4 \\
& & 0.096 & 126 \\
& & 0.020 & 605 \\
\hline \hline
\end{tabular}


The Hencky strain and the strain rate are calculated for the FSR setup as $\varepsilon(t)=-2 \ln \left(D_{\text {mid }}(t) / D_{0}\right)$ and $\dot{\varepsilon}=d \varepsilon / d t$. To ensure a constant strain rate, the mid-diameter is required to decrease exponentially with time during stretching. A close loop control scheme which consists of a feed-forward and a feedback loop [69,70] is employed in the FSR to ensure accurate constant stretch rates. The close loop control scheme is limited by a maximum stretch rate of $5 \mathrm{~s}^{-1}$. For the rates faster than this, an open loop control scheme that switches off the feedback contribution has to be used. In the latter case, the feed-forward control parameters, which define the velocity of the top plate, were obtained by trial-and-error until the midplane diameter decreases exponentially with time [71]. Finally, it is important to refer that in both extensional rheometers, CaBER and FSR, the fluid sample was always pinned to the edges of the plates.

\section{RESULTS AND DISCUSSION}

\section{A. Shear rheology}

\section{Linear viscoelasticity-flow curves}

The linear viscoelastic response of colloidal suspensions with similar volume fractions and different particle sizes is presented in Fig. 2. To allow a direct comparison of different systems the elastic and viscous moduli, $G^{\prime}$ and $G^{\prime \prime}$, are normalized by thermal energy density, and the frequency is multiplied by the Brownian time, $P e_{\omega}=\omega t_{B}=\omega 6 \pi \eta_{s} R^{3} / k_{B} T$. The two smaller particle size samples with $\varphi \approx 0.6$ (in the glass regime) show frequency dependent moduli with a solidlike behavior, where $G^{\prime}$ dominates over $G^{\prime \prime}$ at a low $P e$, indicative of the kinetically trapped particles within a cage formed by their neighbors. At high frequencies, $G^{\prime}$ and $G^{\prime \prime}$ approach each other at a characteristic crossover frequency and exhibit a liquidlike behavior with $G^{\prime \prime}>G^{\prime}$ at higher frequencies, a response related with local in-cage particle diffusion. For the larger particle size $(R \approx 689 \mathrm{~nm})$ samples, the high frequency regime liquidlike response due to in-cage motion is seen

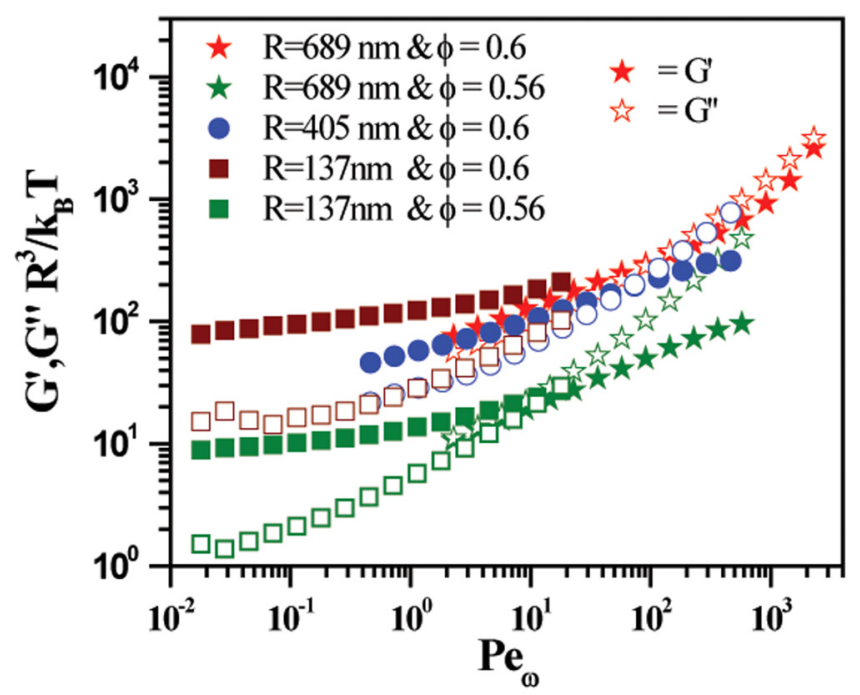

FIG. 2. Dynamic frequency sweeps for different particle sizes as indicated in squalene. Solid symbols represent the elastic moduli, $G^{\prime}$, and open symbols the viscous moduli, $G^{\prime \prime}$, both scaled by $k_{B} T / R^{3}$ versus scale frequency $P e_{\omega}$. better (as $P e_{\omega}$ extends to higher values) both in the glass sample $(\varphi \approx 0.6)$ and the supercooled liquid $(\varphi \approx 0.56)$.

Figure 3(a) shows flow curves for the same systems where the viscosity was measured as a function of shear rate at
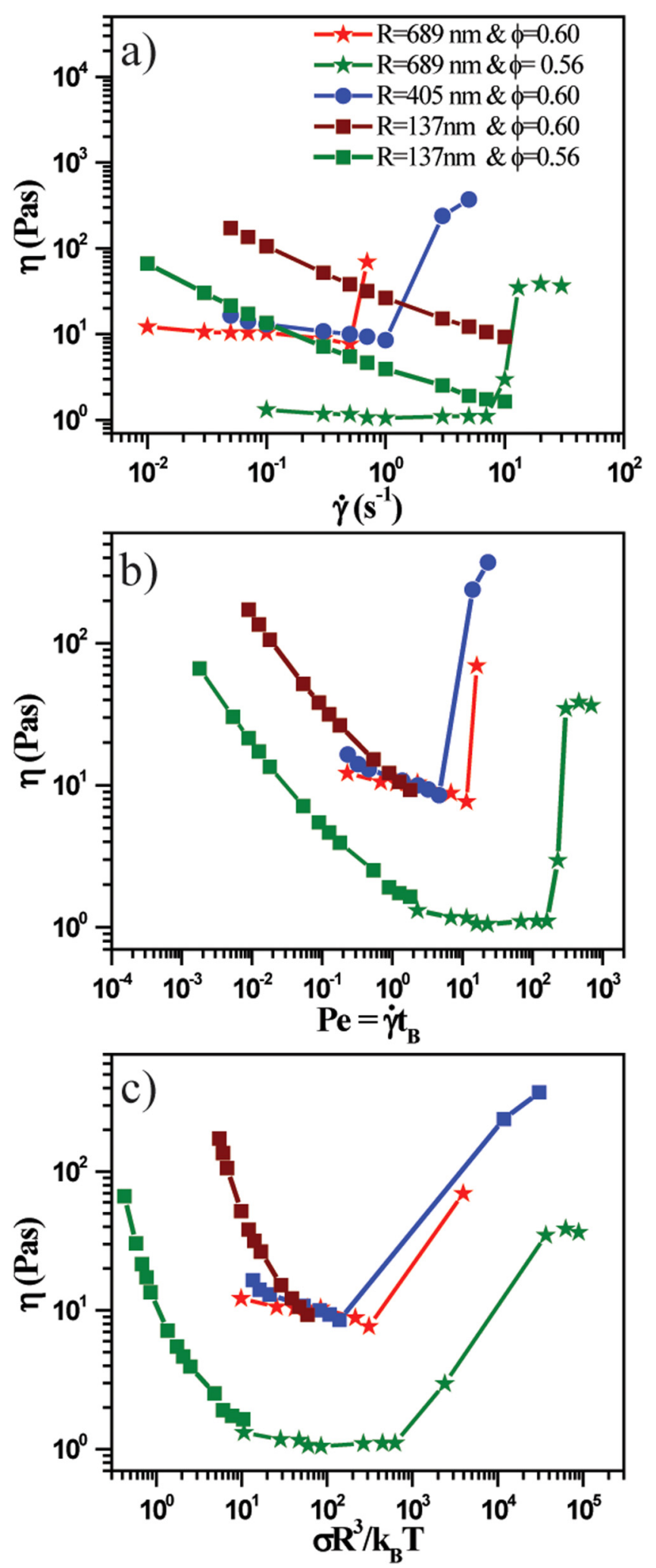

FIG. 3. Flow curves for all samples measured with different particle sizes and volume fractions as indicated (a) transient viscosity plotted as a function of shear rate, (b) plotted as a function of dimensionless $P e$, and (c) as a function of scaled stress. The solid line of slope of 1 indicates the expected dependence for DST. 
transient following step-rate experiments as explained above. With the exemption of the smaller particle $(R \approx 137 \mathrm{~nm})$, all samples show a sharp increase in the viscosity at a critical shear rate, indicating a DST behavior. The critical shear rate for the onset of DST decreases with increasing particle size as expected [61,72]. With the aim to scale out the effect of particle size, the data are also plotted versus the dimensionless shear rate, i.e., $P e$ in Fig. 3(b). More specifically, we detect the onset shear rate of DST at around $0.6 \mathrm{~s}^{-1}$ and $3 \mathrm{~s}^{-1}$ for particles with $R \approx 689 \mathrm{~nm}$ and $R \approx 405 \mathrm{~nm}$, respectively, which correspond to similar values of $P e(\approx 13.72$ and 13.94 , respectively) for the glassy samples of the two larger particles at $\varphi \approx 0.6$. This finding suggests that, at the same volume fraction, a critical $P e$ needs to be exceeded for DST to take place. Note that the sample with the smaller PMMA particles $(R \approx 137 \mathrm{~nm})$, at the same volume fraction $(\varphi \approx 0.6)$, does not show any DST as this critical $P e$ (of the order of 10) has not been reached [see Fig. 3(b)]. This is similar to what is observed in Fig. 2, where for the smaller particle size sample data do not reach the high frequency regime. The critical shear rate for the onset of DST is increasing with decreasing volume fraction as demonstrated by the measurement with the largest particles at $\varphi \approx 0.56$, just below the glass transition. The viscosity is finally plotted in Fig. 3(c), as a function of the scaled stress, $\sigma R^{3} / k_{B} T$, for all different particle sizes and volume fractions. In the shear thickening regime, the viscosity scales almost linearly with the stress indicative of a DST response. Moreover, the onset dimensional shear stress appears to be the same for all samples suggesting that this is the determining quantity as a consequence of osmotic pressure being the dominant interparticle force before shear thickening [73].

\section{Normal stresses}

The first normal stress difference $N_{1}$ measured together with the shear stress shown in the flow curves of Fig. 3, is plotted in Figs. 4(a) and 4(b), as a function of shear rate and $P e$, respectively, similarly with the representation of shear stress in Fig. 3. As mentioned, in Sec. II B, for samples where strong shear thickening and dilatancy effects are observed, the transient $N_{1}$ data shown in Fig. 4 (as well as viscosity data of Fig. 3) were determined before the onset of dilatation (see Figs. 6 and 7). For all samples up to the critical shear rate where shear-thickening phenomena set-in, we observe $N_{1} \approx 0 \mathrm{~Pa}$, suggesting relatively weak structural changes, as it was seen in Fig. 3 and discussed above. For the two larger particles $(R=689 \mathrm{~nm}$ and $R=405 \mathrm{~nm})$ at the higher volume fraction $(\varphi=0.6)$ measured, $N_{1}$ increases suddenly around the critical shear rate where shear thickening is observed, following the behavior of the shear stress (Fig. 3). For the larger particles $(R=689 \mathrm{~nm})$ at lowest volume fraction though $(\varphi=0.56), N_{1}$ starts acquiring negative values at rates much lower (around $1 \mathrm{~s}^{-1}$ ) that the onset of shear thickening $\left(10 \mathrm{~s}^{-1}\right)$ and keeps decreasing significantly well in the shear thickening regime.

\section{Transient response (start-up shear)}

Figure 5 presents the measured stress versus strain during start-up shear tests at different shear rates for all three samples. For the samples presented in Figs. 3(a) and 3(b), we can observe a pronounced stress overshoot at high shear rates. In general, in colloidal suspensions and glasses the stress overshoot is related to structural deformation of the first neighbor cage as stress is stored during an initial deformation stage and then a release upon cage breakage and flow $[74,75]$. This takes place at strains that can be identified as the yield strain which for HS glasses is of the order of $10 \%$, with a weak nonmonotonic volume fraction dependence and a decrease towards zero at random close packing. In the experiments however shown in Fig. 5 the stress overshoot is detected at much larger strains (of the order of 100\%) indicating that they are of a different origin and not related with yielding but rather with larger length-scale rearrangements. As this take place only for systems and shear rates only where shear thickening is observed (Figs. 3 and 4), they must reflect the onset of such phenomena during start-up shear. Moreover, they appear rather complex with the second peak observed after the first. Note that the second peak seems to
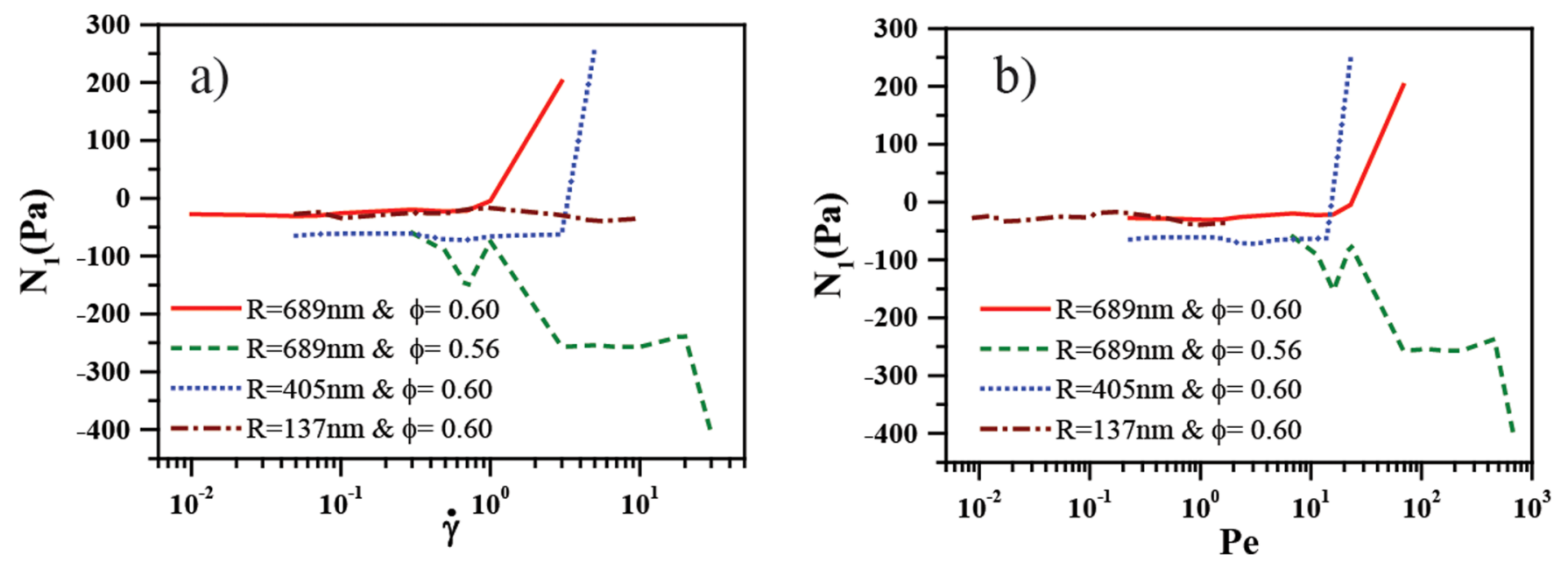

FIG. 4. (a) First normal stress difference, $N_{1}$, as a function of shear rate for all samples measured as indicated. (b) The same data as in (a) plotted versus the dimensionless shear rate, $P e$. 

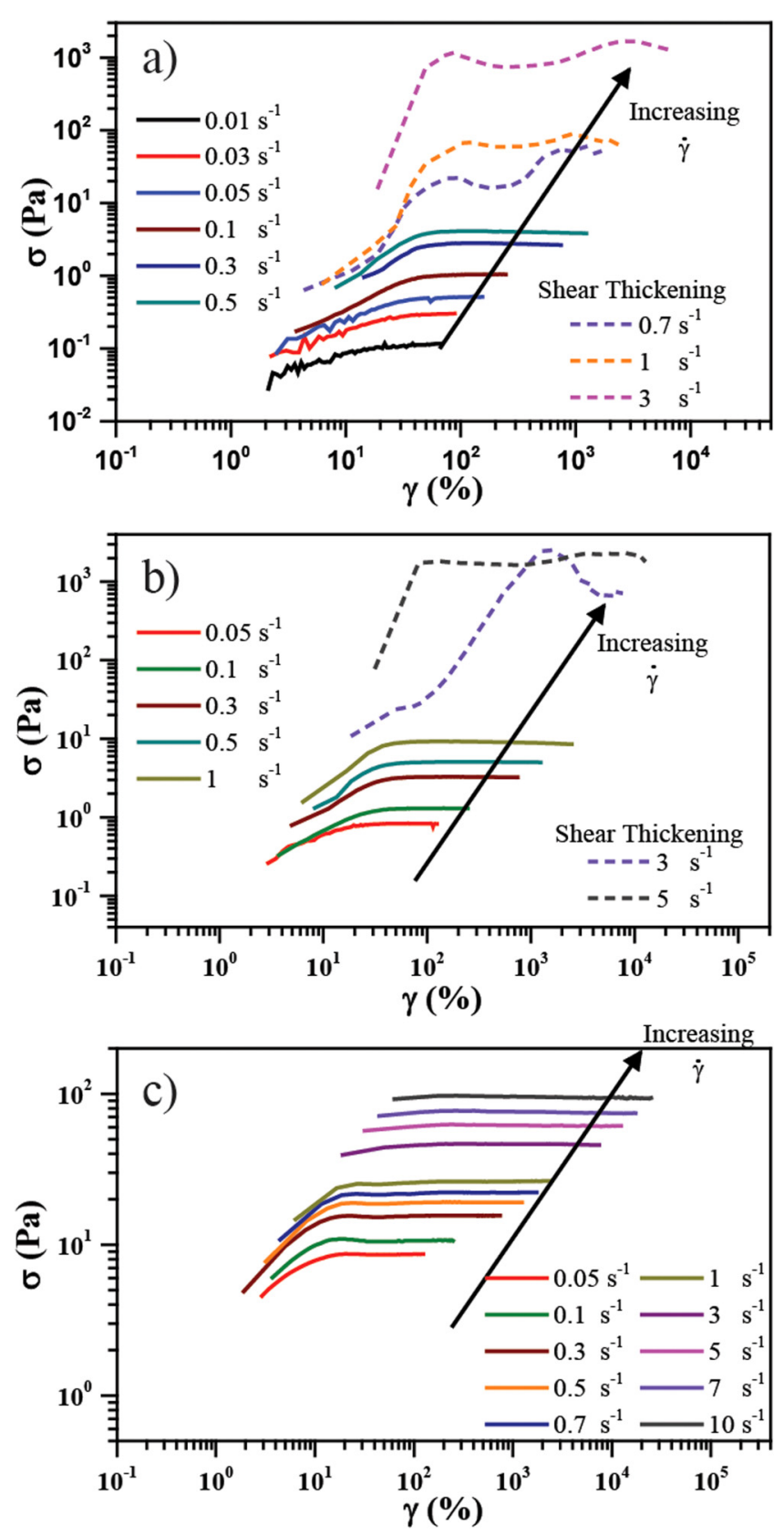

FIG. 5. Step-rate experiments (stress versus strain) at different shear rates for all samples with different particles sizes; large particles $R \approx 689 \mathrm{~nm}$ (a), intermediate size particles, and $R \approx 405 \mathrm{~nm}$ (b) and small particles, and $R \approx 137 \mathrm{~nm}$ (c). All samples were measured at a volume fraction of $\varphi \approx 0.60$.

be related with flow instabilities, as it will be discussed below in Sec. III A 4. The onset shear rate for the overshoot observation is similar with the critical shear rate, where the shear viscosity exhibits a sharp increase, i.e., the onset of DST (Fig. 3). Moreover, the sample with the smallest particles [Fig. 5(c)] does not exhibit any such stress overshoot, in agreement with observations in Fig. 2(b) where shear thickening is absent for this sample in the experimental shear rate window.

The normal stress $\sigma_{N}$ is shown in Fig. 6 during transient start-up shear experiments for the sample with the two larger particles at $\varphi \approx 0.6$ [corresponding to stress data shown in Figs. 5(a) and 5(b)]. For shear rates below the onset of DST, $\sigma_{N}$ remains constant and nearly zero. As the shear rate is increased and approaches the vicinity of shear thickening, however, we observe the development of a negative $\sigma_{N}$ at high strain values (Fig. 4), with a minimum near the second stress overshoot and a subsequent increase toward zero at higher strains. This behavior observed for $\dot{\gamma}=0.7$ and $1 \mathrm{~s}^{-1}$ for particles with $R \approx 689 \mathrm{~nm}$ and $\dot{\gamma}=3 \mathrm{~s}^{-1}$ for particles with $R \approx 405 \mathrm{~nm}$. The negative $\sigma_{N}$ values indicate that contributions from lubrications forces become dominant as detected in previous studies [50,51]. Therefore, under such conditions, particle collisions may lead to aggregates as a result of short-range hydrodynamic forces, overcoming due to the shear flow of the repulsive forces between particles and consequently the lubrication film.

At the highest shear rates measured however, for both particles shown in Fig. 6, we detect first an increase in $\sigma_{N}$ to positive values in the vicinity of the first stress overshoot and subsequently a drop to negative values around strains where the second stress overshoot is detected, as seen at lower shear rates. The transition from $\sigma_{N}>0$, observed during start-up shear at high rates around the first stress overshoot which is due to shear thickening and underlying frictional interactions between particles [54] around the first stress overshoot, to $\sigma_{N}<0$ at higher strains around and beyond the second stress overshoot is an artifact due to dilatancy accompanied stickslip behavior and flow instabilities as will be discussed below (see Fig. 7 in Sec. III A 4).

\section{Visual observation}

We complement the rheology data with the direct observation of the sample during shear. The edge of the sample was monitored via a video camera (Multimedia view) during the step rate experiments in order to detect any macroscopic morphological changes related with shear thickening as the strain increases. As at high volume fractions trimming of the excess sample in the gap is a difficult, we leave some excess sample around the cone as seen in the images of Fig. 7. Although similar experiments in shear thickening cornstarch suspensions [30] have shown that excess fluid around the geometry may affect the rheological response, as boundary conditions change, we have not observed any clear effect in our measurements; however, it should be noted that our experiments were performed in a cone-plate geometry, rather than in a plate-plate with a varying gap. Along these lines, we also performed measurements with a cone-partition-plate (CPP) geometry, which is often used in polymer melts to suppress edge fracture effects at high shear rates [76]. As shown in the supplementary material [77], the onset of shear thickening both in steady shear measurements as well as in oscillatory shear was not affected by the use of the CPP. Although some details of the curves are different, no significant changes were detected with the use of the CPP.

Figure 7(a) shows the HS glass with the larger particle size, $R \approx 689 \mathrm{~nm}$ (with $\varphi=0.6$ ), at $\dot{\gamma}=3 \mathrm{~s}^{-1}$ (or $P e \approx 68.61$ ), a value which is above the critical value where DST is detected. The evolution of the shear stress and the normal stress $\sigma_{N}$ are shown during the start-up shear test as a function of strain along with corresponding images of the sample edge. The shear stress is increasing until point B, where the 

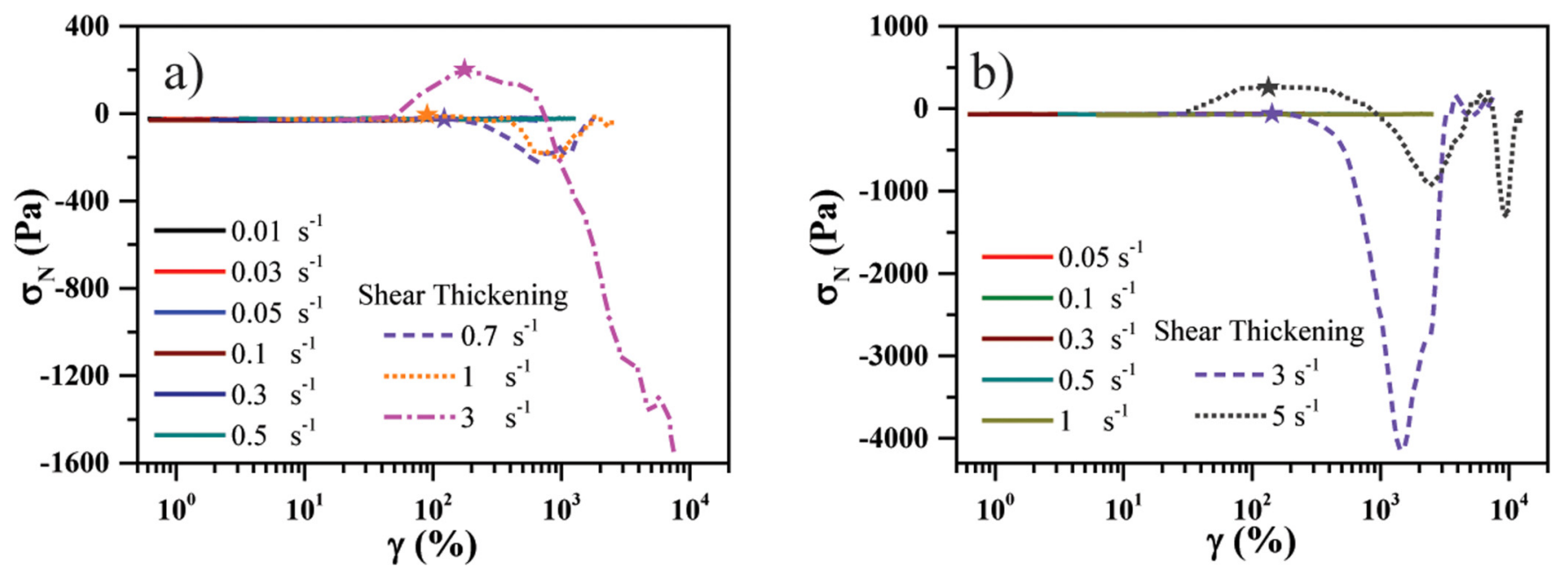

FIG. 6. Normal stress $\sigma_{N}=2 \boldsymbol{F}_{N} / \pi r^{2}$ during transient start-up shear experiment versus strain, $\gamma$, for particles with $R \approx 689 \mathrm{~nm}$ (a) and $R \approx 405 \mathrm{~nm}$ (b). All samples were measured at a volume fraction of $\varphi \approx 0.60$. The asterisks denote the point in time (or strain), where the effective viscosity data plotted in Fig. 4 were determined.

first stress overshoot at strains above $100 \%$, related with DST, is seen as mentioned above. At this point, we detect a slight increase in $\sigma_{N}$ (with positive values) which continues until point $C$. Here, we visually observe that the surface of the sample turns opaque and rough, a signature of dilatation of the material. This response at high shear rates can be understood as follows: HS glass starts to flow under the application of a constant shear rate after the yield strain (typically of the order of $10 \%$ in the glassy regime) is exceeded, as shown in Fig. 5 for low rates and demonstrated in previous studies [74,78]. At high shear rates though, where the stress cannot be detected in the linear regime, i.e., at early times and low strains, the sample shear thickens, and a further stress increase and overshoot is observed at strains above $100 \%$. The stress overshoot is then accompanied by a slight increase in $\sigma_{N}$ which suggests the lubrication film between the particles is overcome by the exerted stress leading to direct contacts between the particles. Since the system continues to be under a constant shear rate, it expands and dilates in order to allow flow to occur along with some stress/energy release. Beyond point $\mathrm{C}$, a second stress overshoot is detected [see also Fig. 5(a)], and the behavior is rather erratic as slip and instabilities set in as seen by the direct visualization of images D and E in Fig. 7(a). We should also note that, after flow cessation, the sample exhibits a rather quick healing in a very short time span of the order of $1 \mathrm{~s}$ as is shown, for the case of the larger particle size in Fig. 7(a).

A similar, but not identical, response was observed in glasses (with $\varphi \approx 0.6$ ) of the intermediate size particles, $R \approx 405 \mathrm{~nm}$. For the same shear rate as the one shown for the larger size particles, i.e., at $\dot{\gamma}=3 \mathrm{~s}^{-1}$ (or $P e \approx 12.15$ ) the stress overshoot takes place when higher strains (more than $1000 \%$ ) are reached [see Fig. 7(b)]. Here, the shear thickening related stress overshoot takes place at point $\mathrm{F}$, where slip and instabilities develop. In this case, $\sigma_{N}$ stays around zero and, turns into negative values as shear thickening occurs, indicating that hydrodynamic forces are predominant in the vicinity of DST [50,51]. At higher strains [points F to I in Fig. 7(b)], past the shear stress overshoot, the normal force increase rise from negative to around zero possibly indicating a transition to a regime where contact forces are also present. For the smaller particles, $R \approx 405 \mathrm{~nm}$, at higher shear rates, $\dot{\gamma}=5 \mathrm{~s}^{-1}$ (corresponding to $P e \approx 20.25$ ) we observe, similarly with the response in the larger particles shown in Fig. 7(a), a stress overshoot at strains around $100 \%$, that is accompanied by a weak increase in the (positive) normal forces in the regime where, the sample exhibits a dilatation [see Fig. 7(c)]. Again, as the strain increases further, $\sigma_{N}$ drops to negative values and exhibits large fluctuations while the stress exhibiting a second overshoot, in a regime where visual observation reveals wall slip and edge instability phenomena. As the suspension is sheared at very large strains, during the transient measurements, edge instabilities and slip also yield negative $\sigma_{N}$, as seen in Figs. 6 and 7. Normal stresses are, therefore, a function of time in this regime, exhibiting fluctuations and where the sign and magnitude are a function of time. On the other hand, the transient values in Fig. 4, determined at the onset of shear thickening (indicated by a star in Fig. 6), do not show any drop of $N_{1}$ for particle sizes, $R \approx 689 \mathrm{~nm}$ and $R \approx 405 \mathrm{~nm}$ at $\varphi \approx 0.6$. In these cases, the normal stress values were extracted at strains just before dilation sets in and before edge instabilities and slip start becoming prominent and affect the normal stress values.

These observations suggest an interplay of hydrodynamic interactions and frictional contacts takes place at dense colloidal suspensions depending on the size and shear rate applied, and related with macroscopic phenomena such as dilatation, wall slip, and edge fracture. Such a behavior is important from the rheological point of view, as was also demonstrated recently in [79] for dense non-Brownian suspensions. It should be noted that this phenomenon is not observed with the smallest particle $(R \approx 137 \mathrm{~nm})$ system mostly due to the limited experimental shear rate window, not reaching the critical $\mathrm{Pe}$ needed. In addition, we cannot exclude the fact that the smaller particle is (proportionally) slightly softer as the stabilization layer is always $\sim 10 \mathrm{~nm}$, and thus larger in proportion to the particle radius for smaller particles. Previous work [80] explicitly shows the stability of both small and large PMMA 
a)

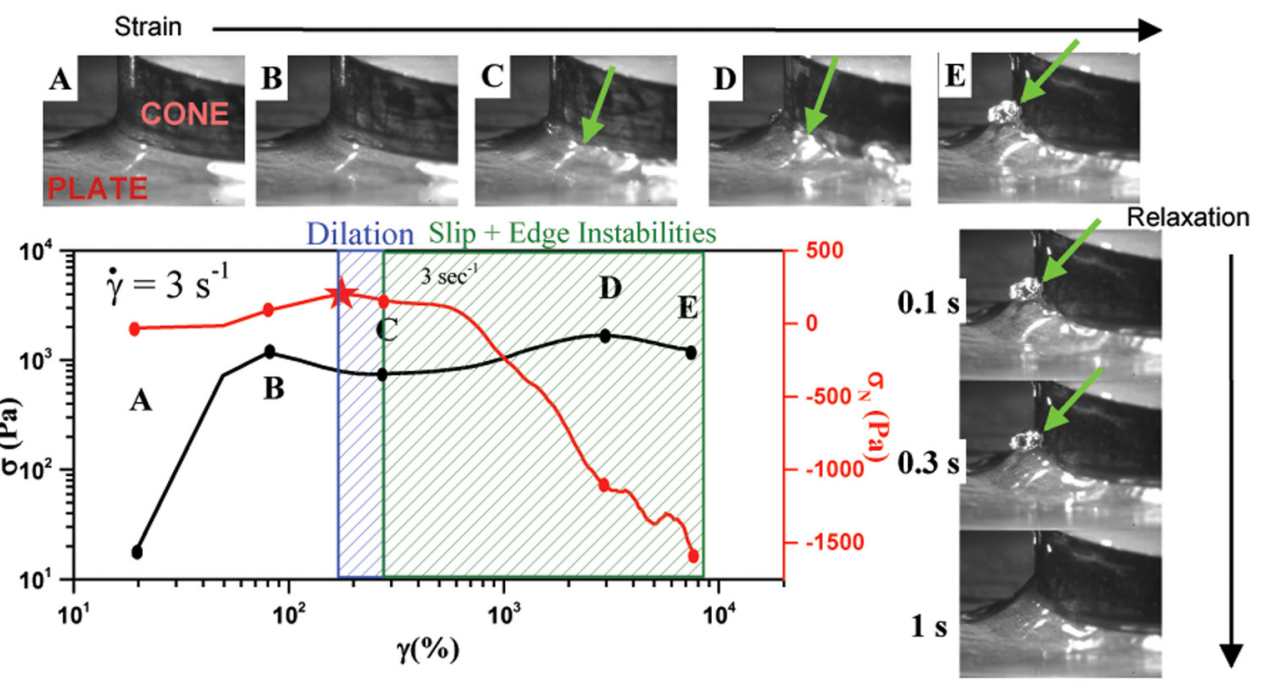

b)
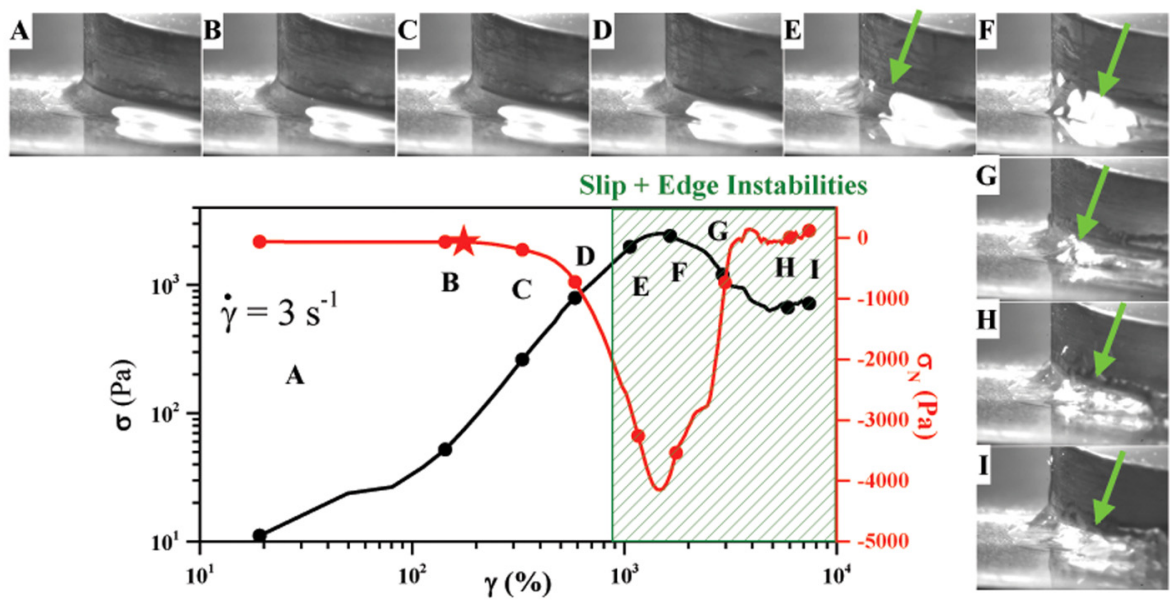

c)
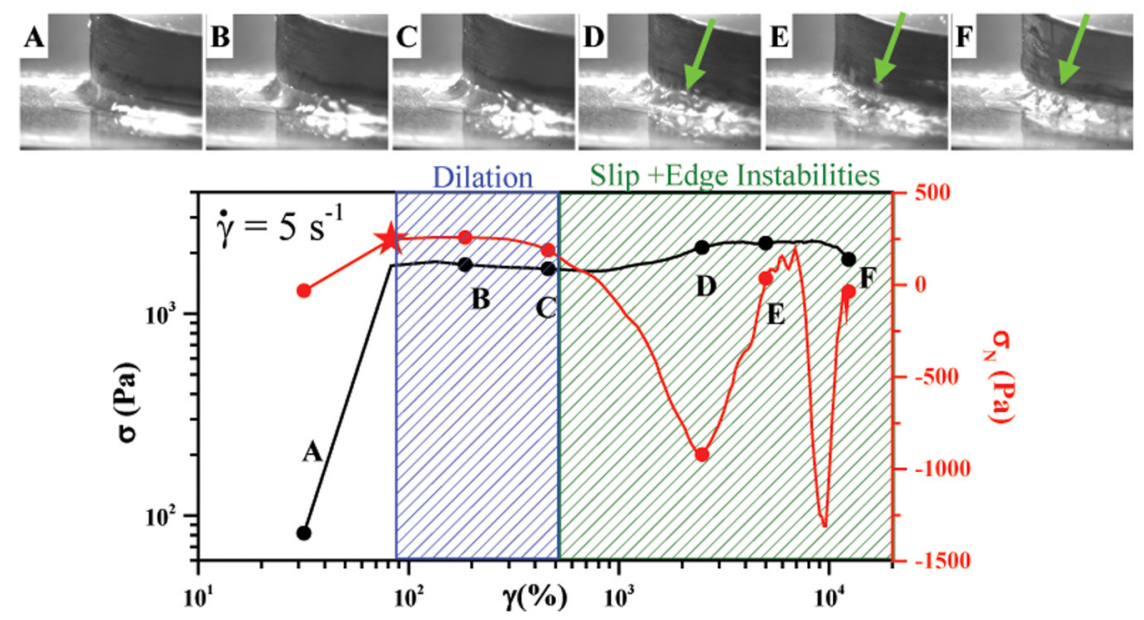

FIG. 7. Stages of step-rate experiments monitored by video recorded images following the stress and normal stress $\sigma_{N}=2 \boldsymbol{F}_{N} / \pi r^{2}$ as a function of strain for: (a) System with large particles, $R=689 \mathrm{~nm}$ at constant shear rate, $\dot{\gamma}=3 \mathrm{~s}^{-1}$; (b) Intermediate size particles, $R=405 \mathrm{~nm}$, at a constant shear rate $\dot{\gamma}=3 \mathrm{~s}^{-1}$ and (c) particles $R=405 \mathrm{~nm}$ at a constant shear rate $\dot{\gamma}=5 \mathrm{~s}^{-1}$. All samples are at a volume fraction of $\varphi=0.60$. In all plots, blue regions denote the observation of dilatation, and green regions slip and edge instabilities as indicated by the green arrows. At indicated points within the step rate experiment images of the sample are shown [(A to E for (a) and (c) and A to I for (b)], while in (a), images of the evolution of the sample after shear is switched off (indicated as relaxation) are also shown at $0.1,0.3$, and $1 \mathrm{~s}$ after shear cessation. Stars indicate (as in Fig. 6) the position in time where the values of the shear and normal stress were determined, prior to the dilatancy. Multimedia views: https://doi.org/10.1122/1.5143653.1; https://doi.org/10.1122/1.5143653.2; https://doi.org/10.1122/ 1.5143653.3. 
a)

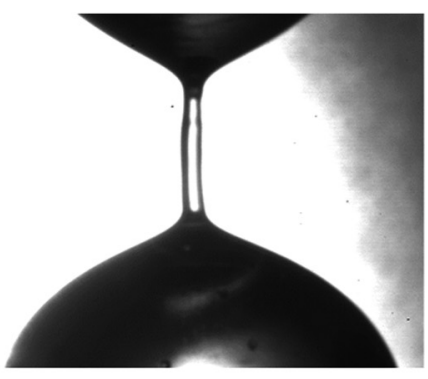

c)

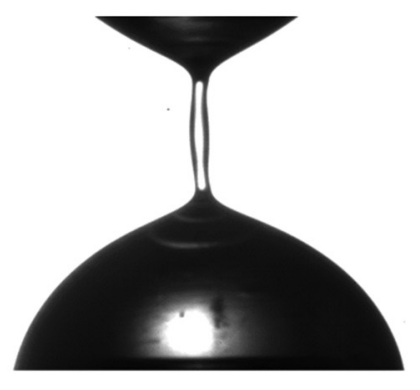

e)

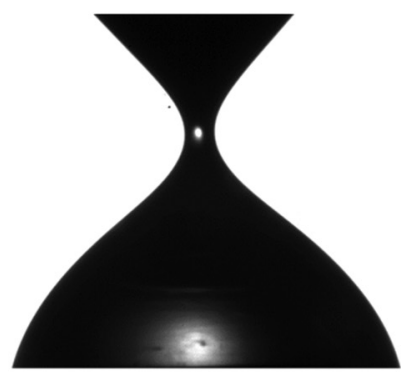

b)

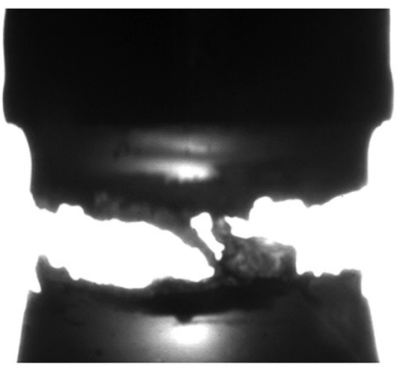

d)

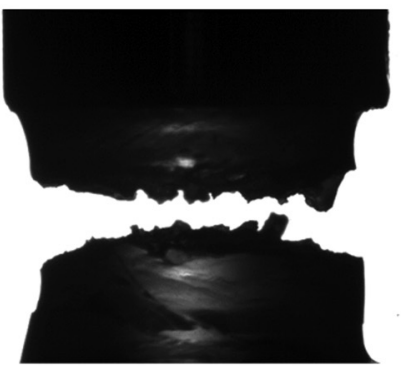

f)

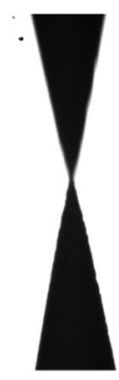

FIG. 8. Final filament shape for HS glasses with particle size $R \approx 689 \mathrm{~nm}$ (top), $R \approx 405 \mathrm{~nm}$ (middle) and $R \approx 137 \mathrm{~nm}$ (bottom) for low ( $\dot{\varepsilon}_{0}<\dot{\varepsilon}_{c}$, left) and high $\left(\dot{\varepsilon}_{0}>\dot{\varepsilon}_{c}\right.$, right) extension rates. All samples are at $\varphi \approx 0.60$. These characteristic filament shapes were obtained with the CaBER set-up.

spheres suspended in squalene with no variation in the bulk viscoelastic modulus observed for as long as $20 \mathrm{~h}$. Moreover, squalene has a refractive index of $(\sim 1.494)$ which is similar to that of PMMA (1.495). Hence, it can be assumed that van der Waals forces have very little effect on the suspension at rest and during flow as stated for other systems [28].

\section{B. Extensional rheology}

We next discuss extensional rheology experiments performed with the same samples, with two different setups, CaBER and FSR.

\section{CaBER experiments}

From the experiments performed with the CaBER setup, we observe two types of responses as shown in Fig. 8. For all samples, similar to shear measurements, there is a critical value of the strain rate, $\dot{\varepsilon}_{c}$, determined by visual observation (Multimedia view) and confirmed by Fig. 11 as we will discuss, below which the sample is stretched uniformly with a filament created by the end of the initial stretching period. At higher rates, $\dot{\varepsilon}_{0}>\dot{\varepsilon}_{c}$, the samples with the two larger particles exhibit a brittle solidlike response manifested by a break-up with an irregular shape during the stretching period. This behavior is shown in Figs. 8(a)-8(d) for all particle sizes, at low (left) and high (right) stretching rates. For the smaller size particle sample, the different behavior at the low and high strain rate regimes is seen in Figs. 8(e) and 8(f); with the high rate response revealing a characteristic pointing shape created during stretching between the two plates [66,81,82].

Similarly, with shear experiments below a critical shear (or strain) rate $\dot{\varepsilon}_{c}$, the surface of the sample remains smooth and the sample is stretched uniformly and symmetrically like a liquid [see Figs. 8(e) and 9(c)]. However, for strain rates higher than $\dot{\varepsilon}_{c}$, the sample exhibits a solidlike response, losing the axial symmetry as they are stretched and exhibiting an irregular shape of the filament as shown in Figs. 9(a) and 9 (b). In the case of solidlike brittle response, the surface texture becomes rough, exhibiting granulation as seen before [59], indicative of microstructural changes in the bulk of the sample. For $\dot{\varepsilon}_{0}<\dot{\varepsilon}_{c}$, samples remain bridging the top and bottom endplates at the end of the stretching zone. Once stretching is completed and the plates are kept constant at their final position, the surface tension causes filament thinning until the point where the filament breaks. On the other hand, for $\dot{\varepsilon}_{0}>\dot{\varepsilon}_{c}$, the fluid solidifies at early stages within the stretching zone and the filament breaks before the top endplate reaches its final position by means of the combined action of pulling forces and surface tension. This behavior is seen in Figs. 9(a) and 9(b) for the two larger particles, in all but the smallest strain rates with the value of $\dot{\varepsilon}_{c}$ decreasing with increasing particle size. 
a)

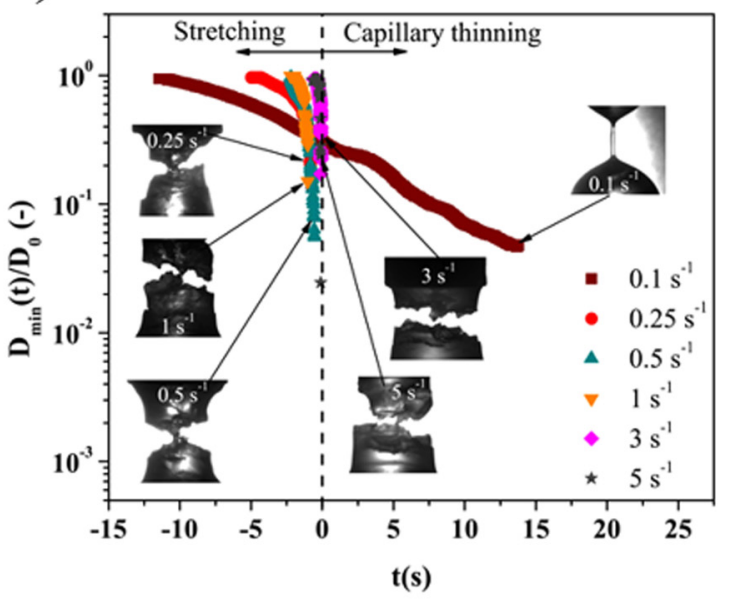

b)

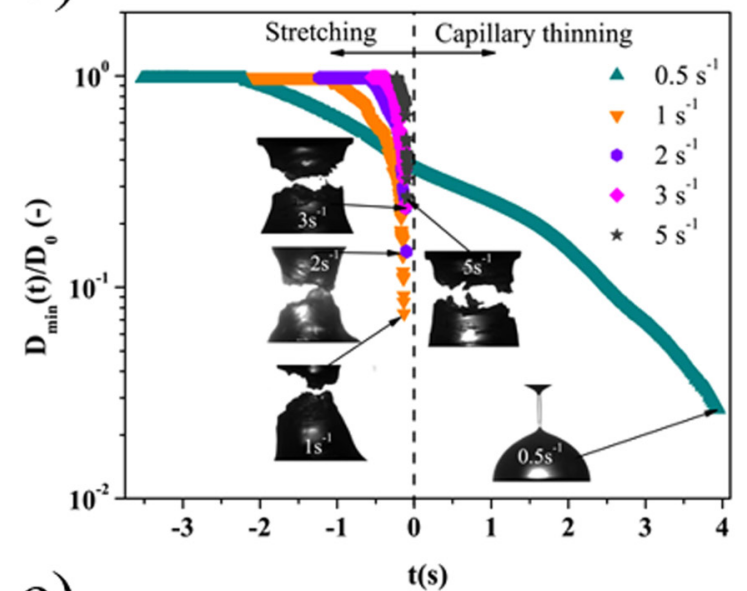

c)

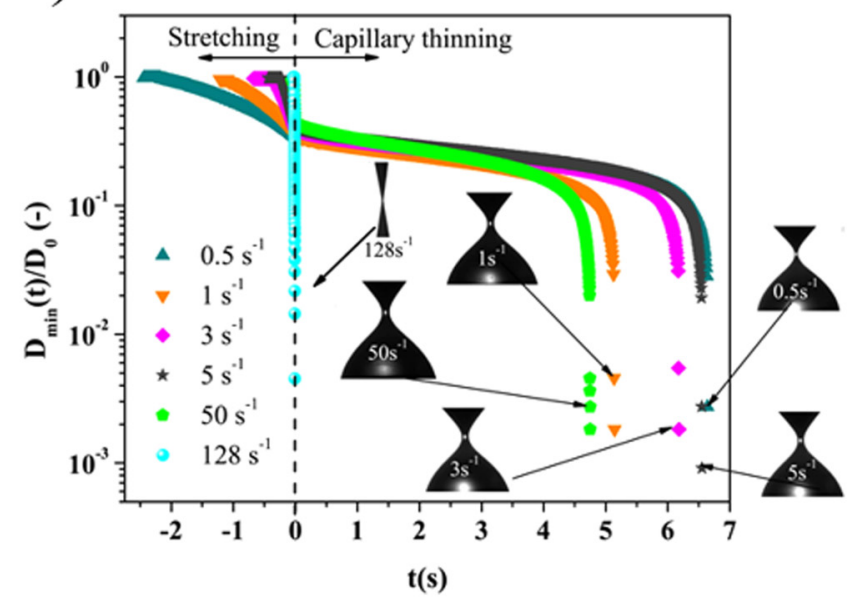

FIG. 9. CaBER setup experiments: comparison between the time evolution of $D_{\min }$ at different samples, and different initial strain rate rates, $\dot{\varepsilon}_{0}$, as indicated, for: (a) sample with particle radius $R \approx 689 \mathrm{~nm}$, (b) for particles with $R \approx 405 \mathrm{~nm}$, and (c) for particles with $R \approx 137 \mathrm{~nm}$. All samples were at a volume fraction of $\varphi \approx 0.60$. Multimedia views: https://doi.org/10.1122/ 1.5143653.4; https://doi.org/10.1122/1.5143653.5; https://doi.org/10.1122/ 1.5143653.6; https://doi.org/10.1122/1.5143653.7; https://doi.org/10.1122/ 1.5143653.8; https://doi.org/10.1122/1.5143653.9.

For the two larger particle sizes $(R \approx 405$ and $R \approx 689 \mathrm{~nm}$ ), we see a filament stretching and breakup as a viscoelastic liquid only at the lowest strain rate measured [Figs. 9(a) and 9(b)]. This viscoelastic behavior at $\dot{\varepsilon}_{0}<\dot{\varepsilon}_{c}$ is combined with a filament recoil at the end when the filament breaks. In Figs. 9(a) and 9(b), we can observe a difference in filament dynamics for $\dot{\varepsilon}_{0}<\dot{\varepsilon}_{c}$, between particles with $R \approx 405$ and $R \approx 689 \mathrm{~nm}$, with the filament thinning occurring faster in suspension with particles of $R \approx 405 \mathrm{~nm}$. Actually for suspensions with the larger particles $(R \approx 689 \mathrm{~nm})$, the filament seems to decelerate at the end, which is usually associated with an increase of the first normal stress difference in the filament as it was reported for liquid jets [83] and demonstrated recently for non-Brownian shear thickening fluids [84]. This is another demonstration of the particle size dependence on the onset of jamming under extensional flow. For the smaller particle size $(R \approx 137 \mathrm{~nm})$, the final filament breaks as in a Newtonian liquid for $\dot{\varepsilon}_{0}<\dot{\varepsilon}_{c}$ [Fig. 8(e)], and thinning dynamics is determined by the Newtonian behavior of the suspending fluid, as it has been reported before $[81,85,86]$.

For $\dot{\varepsilon}_{0}>\dot{\varepsilon}_{c}$, samples with the two larger particles ( $R \approx 405 \mathrm{~nm}$ and $R \approx 689 \mathrm{~nm}$ ) exhibit a brittle failure reminiscent to crystalline solid materials. Under these conditions, the samples fracture without any apparent yielding and plastic deformation observed [Figs. 8(b) and 8(d)]. Experiments with the smallest particles $(R \approx 137 \mathrm{~nm})$, however, reveal a highly ductile behavior under elongation, with the sample flowing until it necks, due to plastic deformation allowed in the microstructure [Fig. 8(f)]. Analogous to the failure mechanics of solid materials [87], there is a critical particle size, $R_{c}$, at which a "nil-ductility" transition from ductile (particles smaller than $R_{c}$ ) to brittle failure will take place. In jammed suspensions of large particles, the reduced local mobility of the particles does not allow any microscopic rearrangements under elongation leading to brittle fracture at high stresses (or strain rates). On the other hand, similar stresses (or rates) induce a ductile failure in a sample with the smallest particles, as local particle mobility is higher (represented by shorter Brownian times). In fact, the ductile failure observed for the smallest particles $(R \approx 137 \mathrm{~nm})$ is represented by filament shapes resembling those detected in a variety of yield stress fluids which exhibit similar break-up shapes, although they do not necessarily have the same microstructure [88-93]. On the other hand, at large strain rates $\dot{\varepsilon}_{0} \gg \dot{\varepsilon}_{c}$, promote brittleness with a horizontal, but irregular fracture lines (see Figs. 8 and 9), similar to what is expected in solid mechanics [87]. Smith et al. [59] also observed similar behavior for a system with similar PMMA particles in octadecene and suggested that this could be the indication of the onset of jamming due to the self-filtration [94] of the system. This is caused at the microscopic level by a differential motion between the particles and the fluid so that particles are left behind in the same position as the fluid drains during the filament thinning, as an effect of the combined force exerted on the sample by the moving plates and surface tension. This mechanism introduces heterogeneities in a particle volume fraction during flow, which influences the dynamics of the filament during the thinning process.

\section{FSR experiments}

Filament stretching measurements were also performed by an FSR setup with the aim to monitor the stress as a function 
of deformation in these HS colloidal glasses. For the smaller particle samples $(R \approx 137 \mathrm{~nm})$, a liquidlike behavior was always seen in the range of the equipment (maximum strain rate of $20 \mathrm{~s}^{-1}$ ). For samples with particle sizes $R \approx 405 \mathrm{~nm}$ and $R \approx 689 \mathrm{~nm}$, we find again a critical strain rate, $\dot{\varepsilon}_{c}$ above which the filament structure changes and the sample shows a solidlike response with an irregular shape and rough texture due to dilatancy as shown in Fig. 1(b), similar to CaBER experiments (Figs. 8 and 9). However, due to the difficulty to perform measurements using the close loop control, since the filament shape is irregular, all measurements for $\dot{\varepsilon}_{0}>\dot{\varepsilon}_{c}$ were performed without it. Therefore, the equipment applied a constant strain rate controlling the top plate velocity [67], by checking the mid-filament diameter as a function of time. The critical strain rate $\dot{\varepsilon}_{c}$ is about $3.0 \mathrm{~s}^{-1}$ (corresponding to $P e \approx 6.5)$ and $0.2 \mathrm{~s}^{-1}(P e \approx 4.5)$ for particles with $R \approx 405 \mathrm{~nm}$ and $R \approx 689 \mathrm{~nm}$, respectively. Considering these values, it should be noted that the strain rate measured in the FSR is deduced using the filament mid-point diameter, while the strain rate in the CaBER is estimated from the velocity of the moving plates. Hence, we find that the critical strain rates $\dot{\varepsilon}_{c}$ in the CaBER is around $0.5 \mathrm{~s}^{-1}(P e \approx 2.3)$ and $0.1 \mathrm{~s}^{-1}$ $(P e \approx 2.3)$ for the HS glasses with particle size of $R \approx 405 \mathrm{~nm}$ and $R \approx 689 \mathrm{~nm}$, respectively. The lower values of the critical rate in the CaBER can be justified not only by the difference in the method, but also by the fact, that in FSR measurements, the mid-filament plane was measured by a laser although jamming and necking be localized in a neighboring plane (where the deformation/stress is different), while CaBER data were deduced at $D_{\text {min }}$ (and not at $D_{\text {mid }}$ ) from high speed camera images that provide more accurate profiling of the filament [see Fig. 1(a)]. Moreover, the stress and the critical rate may change if the initial sample size (aspect ratio) is varied; in this work, we kept the initial sample size the same.

Our main aim however in using the FSR was to obtain complementary information by measuring the stress during extensional flow and jamming of HS colloidal glasses. In Fig. 10(a) (top), we show the measured force from an FSR setup for strain rates below the critical rate for the observation of a solidlike response as a function of Henky strain, for the three different particle size systems as indicated. For such low rates (i.e., for $\dot{\varepsilon}_{0}<\dot{\varepsilon}_{c}$ ), the measured forces are rather small, and the data are quite scattered for all three samples measured; therefore, no quantitative estimation of the stress during the extensional shear is in practice possible.

For high rates however, at $\dot{\varepsilon}_{0}>\dot{\varepsilon}_{c}$, samples show the solidlike response seen with the CaBER setup with the stretched filament's surface turning rough and opaque, while the sample thins irregularly and eventually granulating and breaking as shown in Fig. 10(b) (top). In these cases, the force is much higher than at low strain rates and clearly detectable by the instrument. Then, the engineering stress, defined as $\sigma_{\text {eng }}=4 F(t) /\left(\pi D_{0}^{2}\right)$, can be determined and plotted [Fig. 10(b)] as a function of Hencky strain. Although the quantitative reproducibility is weak, qualitatively different measurements show the same features. At this high strain rate regime, both samples shown in Fig. 10(b) show a stress increase with deformation and exhibit a stress peak at a

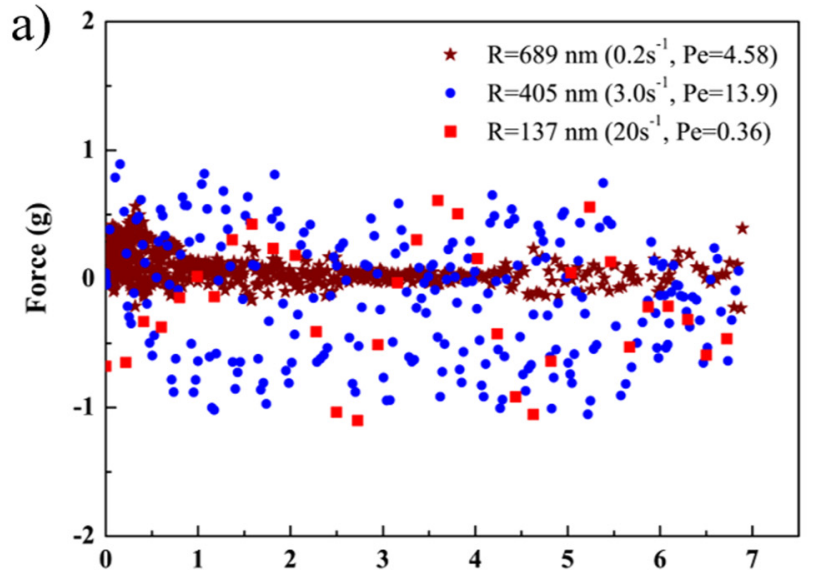

b)

Hencky Strain (-)

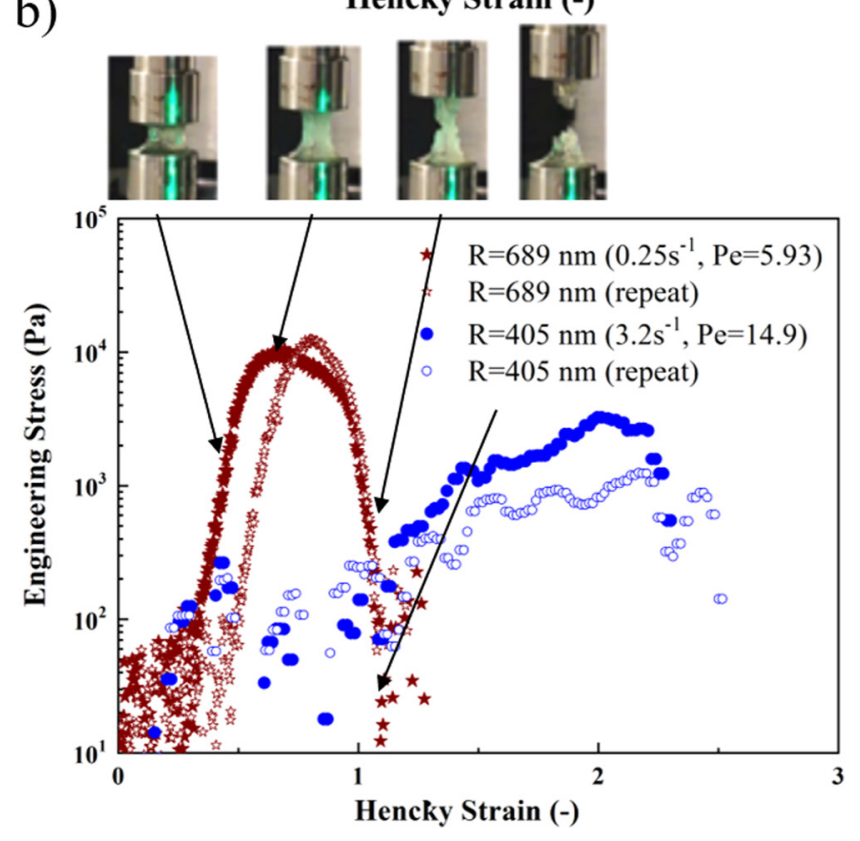

FIG. 10. Experiments in a FSR extensional setup for low (a) and high strain rates (b). The measured force versus the Hencky strain for all samples at $\dot{\varepsilon}_{0}<\dot{\varepsilon}_{c}$ are shown in (a) similarly, in (b) we show the measured engineering stress versus the Hencky strain at $\dot{\varepsilon}_{0}>\dot{\varepsilon}_{c}$ together with the corresponding images of the sample at different instances during stretching, as indicated by the arrows.

certain Hencky strain. Correlating the stress response with simultaneous imaging of the sample, we see that the stress increase, indicative of strain-hardening of the sample, takes place while the sample is still connected to the two plates while as the filament starts to exhibit necking and break the stress starts to decay till it drops to zero when the filament is fully broken.

As has been already reported [95] in weakly strainhardening fluids the engineering stress under extensional flow passes through a maximum when the elastic filament is unstable and starts necking, but grows without bounds at larger values of Deborah number $(D e)$. Analogous results have been published for shear thickening fluids by Rothstein and co-workers [57,58], which reported that just prior to the onset of filament failure, the extensional viscosity, which is proportional to the engineering stress measured in the FiSER, tends to go through a maximum. In the case of shear 
thickening fluids consisting of fumed silica particles with fractal chainlike structures, light scattering measurements showed that the extensional hardening was due to the alignment of nanoparticles and the formation of long strings of aggregates in the flow direction [58]. For cornstarch particles, which are nearly symmetric, the speed and magnitude of strain hardening increase remarkably fast with an extension rate. At low extension rates, the fluid exhibits a Newtonian response, while at moderate extension rates, it shows a weak strain hardening and forms long coherent fluid filaments. At a critical extension rate however, a dramatic increase in both the rate and magnitude of the strain hardening was observed with an increasing extensional rate. Such a dramatic increase in strain hardening was attributed to particle aggregation and the formation of an interconnected jammed network of clusters across the filament with a finite maximum strength independent of the extensional rate [57].

Here, the samples with the larger particles $(R \approx 689 \mathrm{~nm})$ seem to exhibit such behavior and develop a stress peak which is more pronounced and take place at lower strains compared to smaller particles $(R \approx 405 \mathrm{~nm})$. These observations indicate the combined effect of the particle size and extension rate on the jamming phenomena due to the agglomeration of the particles and subsequent granulation of the sample suggesting that the mechanism responsible for jamming of concentrated HS suspensions and glasses during extensional flow is similar to that under shear. However, the critical deformation rates for developing such phenomena are lower in extensional than in shear flow. Our data show that, for particles with $R \approx 405 \mathrm{~nm}$ and $R \approx 689 \mathrm{~nm}$, jamming occurs at $P e \approx 13$ during the shear flow and $P e \approx 2.3$ during extensional flow when measured by the CaBER, while when the FSR is used, we get a critical shear rate of $P e \approx 6.5$ and $P e \approx 4.5$ for particles with $R \approx 405 \mathrm{~nm}$ and $R \approx 689 \mathrm{~nm}$, respectively. Although there are differences between CaBER and FSR results, as discussed above, all measurements of the critical $P e$ are clearly lower than those in shear experiments. The reason for this might be associated with confinement effects introduced in extensional flow due to a compressive flow in the radial direction that may increase packing, and subsequently promote local particle aggregation during axial stretching. It should be noted that at low initial aspect ratios $\left(\Lambda_{0}\right)$, there is a contribution in the measured stress that arises from the shear components. As mentioned earlier, this is caused by the no-slip boundary condition at the end plates and is more prominent for samples with small aspect ratios. This effect could be accounted for using an empirical correction factor as suggested by Rasmussen et al. [96] for homogeneous polymer systems. However, we did not use the correction factor here since we clearly have an inhomogeneous system due to a dilatancy of the material. Therefore, the engineering stress presented in Fig. 10(b) may vary for samples with different aspect ratios and may be influenced by the filament shape as well; thus should be used only qualitatively. Our findings clearly suggest that a more systematic investigation of the role of these variables in colloidal suspensions is needed.

In summary, the extensional behavior of HS glasses at a specific volume fraction depends on particle size and applied

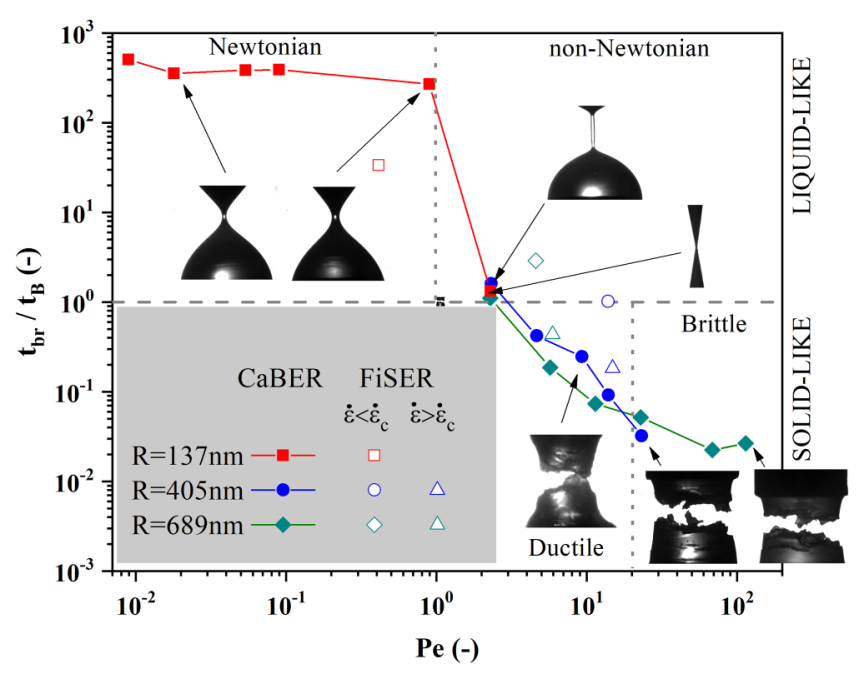

FIG. 11. Dimensionless diagram of the different behavior shown by different samples at $\varphi \approx 0.60$ under elongational flow obtained with the CaBER setup.

extension rate. We can infer that the conditions imposed in the CaBER setup, namely, the initial extension rate $\dot{\varepsilon}_{0}$, determine the arrangement of the particles during flow resulting in filament thinning processes with different shapes and breaking times. Figure 11 shows a master curve in a dimensionless diagram, where the time it takes for the filament to break up since the top plate starts moving from its initial position at $h_{0}$, normalized with the Brownian time $\left(t_{b r} / t_{B}\right)$, is plotted against the Péclet number, $P e=\dot{\varepsilon}_{0} t_{B}$, for extensional experiments in the CaBER setup. This diagram suggests that samples show liquidlike behavior when the characteristic breaking time in the experiment is larger than the Brownian time $\left(t_{b r} / t_{B}>1\right)$ otherwise, the behavior is solidlike. This is very reminiscent of arguments for the onset of shear thickening in HS colloids [28]; the onset stress for shear thickening, $\sigma_{\min }=\eta \dot{\gamma}_{\min }$, related also with an onset shear rate (or $P e$ ), reflecting the fact that an external shear stress must exceed all local stress barriers that prevent relative shear between particles. As long as the extensional deformation is performed slower than stress relaxation induced by local particle diffusion (related with the Brownian time), the sample exhibits a fluid-like response. When this is not the case, the actual filament breakage time becomes smaller than the Brownian time and the sample starts behaving as a jammed granular system manifesting dilatancy and fracture.

The same observation also applies to the FSR experiments suggesting that it is independent of the setup used to induce extensional flow. More interestingly, the diagram indicates the distinction between Newtonian $(P e<1)$ and non-Newtonian $(P e>1)$ behaviors, within the liquidlike regime $\left(t_{b r} / t_{B}>1\right)$ as well as between ductile $(P e<20)$ and brittle response $(\mathrm{Pe}>20)$, within the solidlike regime $\left(t_{b r} / t_{B}<1\right)$. Table II summarizes this behavior and indicates that the critical Péclet number for the onset of granulation in the CaBER is $P e_{c} \approx 2.3$, in the FSR setup is on average $P e_{c} \approx 5$, while in shear experiments, $P e_{c} \approx 13$ (Fig. 4, where $N_{1}>0$ ), independent of the particle size. The fact of having lower critical $P e$ values for the extensional flow than the 
TABLE II. Summary of the different rheological behaviors observed in the dimensionless diagram for the samples at $\varphi \approx 0.60$ under the elongational flow as deduced from CaBER experiments.

\begin{tabular}{lcccc}
\hline \hline$t_{b r} / t_{B}$ & $P e<1$ & $1<P e<2.5$ & $2.5<P e<20$ & $P e>20$ \\
\hline$>1$ (liquidlike) & Newtonian & \multicolumn{2}{c}{ Non-Newtonian } & \\
& & $\begin{array}{c}\text { Viscoelasticlike } \\
\text { Yield-stresslike }\end{array}$ & \\
& & & Jamming & Jamming \\
& & & Ductile & Brittle \\
\hline \hline
\end{tabular}

steady shear is consistent with the flow dynamics associated with each type of experiment. In steady shear flow, the separation of two particles is linear, unlike in extensional flow, in which the separation is exponential as time increases [97].

For an ideal uniaxial extensional and shear experiments, we expect that $P e_{\text {ext }} / P e_{\text {shear }}=1 / \sqrt{3}$, since $\dot{\varepsilon}_{0}=\dot{\gamma} / \sqrt{3}$ [97]. However, as stated above, during the early stages of both the CaBER and the FSR measurements, besides the extension rate, there is an additional contribution from a shear component proportional to $\dot{\varepsilon}_{0} / \Lambda_{0}^{2}$, i.e., $\dot{\gamma}^{*}=k \dot{\varepsilon} / \Lambda_{0}^{2}$. Thus, in the simplest case, that $k=1$, the strain rate tensor in the CaBER and the FSR is

$$
\overline{\bar{\gamma}}=\left(\begin{array}{ccc}
-\dot{\varepsilon}_{0} & \frac{\dot{\varepsilon}_{0}}{\Lambda_{0}^{2}} & 0 \\
\frac{\dot{\varepsilon}_{0}}{\Lambda_{0}^{2}} & -\dot{\varepsilon}_{0} & 0 \\
0 & 0 & 2 \dot{\varepsilon}_{0}
\end{array}\right),
$$

with the magnitude of $\overline{\bar{\gamma}}$ being

$$
\dot{\gamma}_{C a B E R / F S R}=+\sqrt{\frac{1}{2}(\overline{\bar{\gamma}}: \overline{\bar{\gamma}})}=\dot{\varepsilon}_{0} \sqrt{3+\frac{1}{\Lambda_{0}^{4}}} .
$$

According to Eq. (2) and the experimental values of $\Lambda_{0}$ in the two setups, we should then expect that for the CaBER we get, $P e_{c_{-} C a B E R} / P e_{c_{\text {shear }}}=1 / 2.5=0.4$ and for the FSR, $P e_{c_{F S R}} / P e_{c_{\text {shear }}}=1 / 6.8=0.147$, (instead of the ideal $P e_{c_{-} \text {ext }} / P e_{c_{-} \text {shear }}=1 / \sqrt{3}$ ). In view of the above, the experimentally determined critical rates in the CaBER, $P e_{C_{-} C a B E R} / P e_{c_{\text {shear }}}=0.13$ are lower than the expected ratio (0.4), whereas for the FSR the experimental ratio, $P e_{c_{-} F S R} / P e_{c_{\text {shear }}}=0.384$ is clearly higher than the expected value (0.147). This might be either due to a deviation of the true shear contribution present in the two experimental setups from the value estimated based on the $\Lambda_{0}$, term with $k=1$ or to different microstructural mechanism of the HS glasses in shear and extension. With regard to the former, despite the smaller initial aspect ratio $\left(\Lambda_{0}\right)$ in the FSR, the total shear contribution in the flow is apparently more than two times smaller than in the CaBER experiments and three times less than the expected value from Eq. (2). The weaker shear contribution in the FSR may be attributed to the two-step stretching protocol used, where an initial stretch at an extension rate well below the onset of extensional

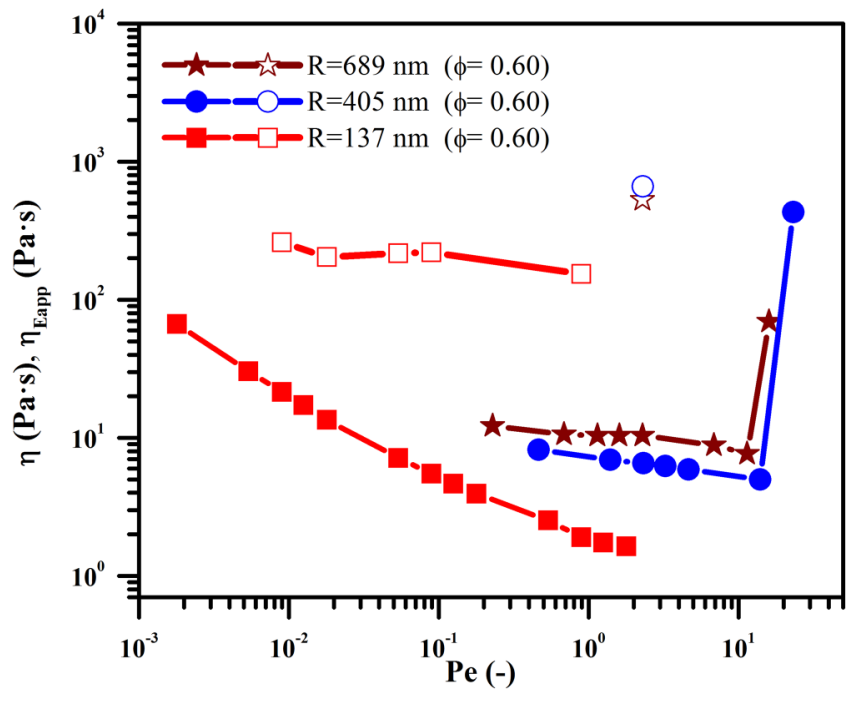

FIG. 12. Comparison between the apparent shear (filled symbols) and extensional (empty symbols) viscosities of the samples at $\varphi \approx 0.6$. Solid lines connect the experimental dots in order to guide the eye.

thickening indeed minimizes the shear effects on the second stretch, which is much faster and imposes a constant strain rate until the filament breaks up. This would quantify to a value of $k_{F S R}=0.37$ assuming that the critical strain rate (for the onset of thickening and dilatation) is the same in extensional and shear experiments, hence attributing the differences in the critical strain rate values in shear and FSR experiments fully to an inaccurate determination of the shear contribution in the latter. A similar approach in the CaBER setup would give $k_{C a B E R}=3.23$ since the shear contribution which is about three times higher than the expected according to Eq. (2). We should note however that these $k$ should be taken cautiously as limiting values that indicate the amount of maximum shear contribution that could be present during the corresponding extensional experiments here.

Finally, the apparent extensional viscosity is also calculated from the CaBER experiments, based on the equation $\eta_{\text {Eapp }}=-\left[\sigma /\left(d D_{\min }(t) / d t\right)\right] \quad[98]$, assuming the surface tension of the solvent as the surface tension of the sample. As it can be observed in Fig. 9, depending on the particle size and the initial extension rate imposed, the filament solidifies and breaks while the top plate is still traveling toward its final position $\left(h_{f}\right)$. When the time to break is larger than the Brownian time, the sample does not solidify while being stretched from $h_{0}$ to $h_{f}$; this holds for $P e<2.5$ (Fig. 11). Therefore, the apparent extensional viscosity can only be calculated once the top plate reaches the final position and the filament thins gradually until it breaks. The results are shown in Fig. 12 as a function of the Péclet number. In all cases, the extensional viscosity is larger than three times the shear viscosity, and therefore the Trouton ratio, $\mathrm{Tr}$, is larger than three even though granulation did not occur yet. This is mostly due to the fact that we are observing a non-Newtonian behavior while $\mathrm{Tr}=3$ normally holds for Newtonian systems. Khandavalli and Rothstein [99] have also presented similar findings, which they attribute to nonspherical or fractal nature of the fumed silica particles used. 


\section{CONCLUSIONS}

We have reported a dilatancy behavior of HS colloidal glasses with different particle sizes under shear and extensional flow. Discontinuous shear thickening and dilatancy is observed with the larger particles at high shear rates, in both volume fractions studied. Start-up shear experiments at different shear rates exhibit stress overshoots above the critical shear rate for the onset of DST, suggesting a change in the suspension microstructure during the flow. Moreover, $N_{1}$ measurements during transient step rate tests have shown a transition from $N_{1} \approx 0$ (at low shear rates) to $N_{1}<1$ (in the vicinity of a critical shear rate) indicating contributions from lubrications forces at lower rates, and to $N_{1}>1$ beyond critical shear rate which is volume fraction and particle size dependent indicating frictional interactions between particles. The critical Péclet number for DST in shear experiments (at $P e_{c r} \approx 15$ ) is independent of the particle size but depends on volume fraction. However, all different samples show approximately the same scaled stress at the onset of DST. Sheared samples were monitored by a video camera, revealing that dilatancy occurs in the presence of contact interactions $\left(N_{1}>0\right)$ in all samples showing DST, i.e., systems with large particles $(R \approx 405 \mathrm{~nm}$ and $R \approx 689 \mathrm{~nm})$ in the glassy state.

Dilatancy for suspensions with particle size of $R \approx 405 \mathrm{~nm}$ and $R \approx 689 \mathrm{~nm}$ was also observed under extensional flow, using both CaBER and FSR setups, where a critical value of the strain rate $\left(\dot{\varepsilon}_{c}\right)$ was detected indicating structural changes in the HS glass. Below a critical strain rate, liquidlike behavior was seen for all particle sizes, with viscoelastic behavior at the filament break for the large particles $(R \approx 405 \mathrm{~nm}$ and $R \approx 689 \mathrm{~nm}$ ) samples, mostly owed to self-filtration effects induced by filament thinning due to surface tension. This mechanism leads to heterogeneities of the particle volume fraction within the sample during flow and is related with differences in filament dynamics in the two different particle size $(R \approx 405 \mathrm{~nm}$ and $R \approx 689 \mathrm{~nm}$ ) samples. The samples with the larger particles $(R \approx 689 \mathrm{~nm})$ exhibit a slowing down of filament thinning, indicating the onset of creation of a jamming network under extensional flow, while for the samples with the smaller particles $(R \approx 137 \mathrm{~nm})$, the filament thinning dynamics is Newtonian-like for $\dot{\varepsilon}_{0}<\dot{\varepsilon}_{c}$. At high strain rates, for $\dot{\varepsilon}_{0}>\dot{\varepsilon}_{c}$, large particle $(R \approx 405 \mathrm{~nm}$ and $R \approx 689 \mathrm{~nm})$ samples exhibit a combination of granulation and brittle fracture. For smaller particles $(R \approx 137 \mathrm{~nm})$, extensional flow at $\dot{\varepsilon}_{0}>\dot{\varepsilon}_{c}$ leads to filament stretching similar to that observed in yield stress fluids which finally break with a ductile type fracture.

These findings were verified by the FSR measurements that similarly show liquidlike behavior for $\dot{\varepsilon}_{0}<\dot{\varepsilon}_{c}$, and solidlike behavior when $\dot{\varepsilon}_{0}>\dot{\varepsilon}_{c}$, both for suspensions with $R \approx 405 \mathrm{~nm}$ and $R \approx 689 \mathrm{~nm}$ particles. Here, jamming, dilatancy, and subsequent granulation were observed in both suspensions, within the range of the equipment (maximum strain rate of $20 \mathrm{~s}^{-1}$ ). Direct observation of stress measurements showed the effect of particle size on jamming and granulation, suggesting that the mechanism governing this behavior under shear is also present in extensional flow. The strain rates involved in these phenomena are lower in extensional flow than in shear flow, mostly due to the confinement effect of the compressive flow in the radial direction that can increase packing, and subsequent the local aggregation of particles during the stretching.

Finally, the Péclet number scales the influence of flow on granulation, for samples with different particle sizes. In shear experiments, this phenomenon was observed above a critical value of $P e_{c r} \approx 15$, while in extensional flow, in the case of CaBER at $P e_{c r} \approx 2.5$, and in FSR at $P e_{c r} \approx 5$. These findings are consistent with the characteristics of each type of flow: the separation of two particles is linear during simple shear flow, unlike in extensional devices where there is an unavoidably combination of extensional and shear flows at the early stage of the experiments, which is larger in the CaBER than in the FSR and results in a much faster structural changes leading to shear thickening.

\section{ACKNOWLEDGMENTS}

The authors would like to express their sincere gratitude to João Maia for valuable and constructive suggestions in this work. Financial support from the EU Project "SmartPro" and National Project ARiSTEIA II "MicroSoft", the Aage og Johanne Louis-Hansen Foundation, Coordenação de Aperfeiçoamento de Pessoal de Nível Superior-Brazil (CAPES)—PRINT No. 88887.310339/2018-00, Fundação de Amparo a Pesquisa de São Paulo (FAPESP) for Grant No. 2012/50259-8, and Fundo Mackenzie de Pesquisa (MackPesquisa, Project No. 181009), is gratefully acknowledged. F.J.G.-R. and L.C.-D. would also like to acknowledge financial support from Fundação para a Ciência e a Tecnologia (FCT), COMPETE and FEDER through Grant Nos. IF/00190/2013 and IF/00148/2013, and Project No. IF/00190/2013/CP1160/CT0003.

\section{REFERENCES}

[1] Barnes, H. A., "Shear thickening ('dilatancy') in suspensions of nonaggregating solid particles dispersed in Newtonian liquids," J. Rheol. 33, 329-366 (1989).

[2] Wagner, N. J., and J. F. Brady, "Shear thickening in colloidal dispersions," Phys. Today 62(10), 27-32 (2009).

[3] Lee, Y. S., E. D. Wetzel, and N. J. Wagner, "The ballistic impact characteristics of Kevlar ${ }^{\circledR}$ woven fabrics impregnated with a colloidal shear thickening fluid," J. Mater. Sci. 38, 2825-2833 (2003).

[4] Galindo-Rosales, F. J., S. Martínez-Aranda, and L. Campo-Deaño, "CorkSTFufluidics-A novel concept for the development of ecofriendly light-weight energy absorbing composites," Mater. Des. 82, 326-334 (2015).

[5] Helber, R., F. Doncker, and R. Bung, "Vibration attenuation by passive stiffness switching mounts," J. Sound Vib. 138, 47-57 (1990).

[6] Laun, H. M., R. Bung, and F. Schmidt, "Rheology of extremely shear thickening polymer dispersions) (passively viscosity switching fluids)," J. Rheol. 35, 999-1034 (1991).

[7] Nilsson, M. A., R. Kulkarni, L. G. Ryan, H. R. Singh, E. Baumhoff, and J. P. Rothstein, "Effect of fluid rheology on enhanced oil recovery in a microfluidic sandstone device," J. Nonnewton. Fluid Mech. 202, 112-119 (2013). 
[8] Hoffman, R. L., "Discontinuous and dilatant viscosity behavior in concentrated suspensions I. Observation of a flow instability," Trans. Soc. Rheol. 16, 155-173 (1972).

[9] Hoffman, R. L., "Discontinuous and dilatant viscosity behavior in concentrated suspensions II. Theory and experimental tests," J. Colloid. Interface Sci. 46, 491-506 (1974).

[10] Hoffman, R. L., "Discontinuous and dilatant viscosity behavior in concentrated suspensions III. Necessary conditions for their occurrence in viscometric flows," Adv. Colloid. Interface Sci. 17, 161-184 (1982).

[11] Egres, R. G., and N. J. Wagner, "The rheology and microstructure of acicular precipitated calcium carbonate colloidal suspensions through the shear thickening transition," J. Rheol. 49, 719-746 (2005).

[12] Egres, R. G., F. Nettesheim, and N. J. Wagner, "Rheo-SANS investigation of acicular-precipitated calcium carbonate colloidal suspensions through the shear thickening transition," J. Rheol. 50, 685-709 (2006).

[13] Brady, J. F., and G. Bossis, "The rheology of concentrated suspensions of spheres in simple shear flow by numerical simulation," J. Fluid Mech. 155, 105-129 (1985).

[14] D'Haene, P., J. Mewis, and G. G. Fuller, "Scattering dichroism measurements of flow-induced structure of a shear thickening suspension," J. Colloid Interface Sci. 156, 350-358 (1993).

[15] Bender, J. W., and N. J. Wagner, "Optical measurement of the contributions of colloidal forces to the rheology of concentrated suspensions," J. Colloid Interface Sci. 172, 171-184 (1995).

[16] Bender, J., and N. J. Wagner, "Reversible shear thickening in monodisperse and bidisperse colloidal dispersions," J. Rheol. 40, 899-916 (1996).

[17] Laun, H. M., and R. Bung, "Rheological and small angle neutron scattering investigation of shear-induced particle structures of concentrated polymer dispersions submitted to plane Poiseuille and Couette flow," J. Rheol. 743-787 (1992).

[18] Maranzano, B. J., and N. J. Wagner, "Flow-small angle neutron scattering measurements of colloidal dispersion microstructure evolution through the shear thickening transition," J. Chem. Phys. 117, 10291-10302 (2002).

[19] Lee, Y. S., and N. J. Wagner, "Rheological properties and small-angle neutron scattering of a shear thickening, nanoparticle dispersion at high shear rates," Ind. Eng. Chem. Res. 45, 7015-7024 (2006).

[20] Kalman, D. P., R. L. Merrill, N. J. Wagner, and E. D. Wetzel, "Effect of particle hardness on the penetration behavior of fabrics intercalated with dry particles and concentrated particle fluid suspensions," ACS Appl. Mater. Interfaces 1, 2602-2612 (2009).

[21] Cheng, X., J. H. McCoy, J. N. Israelachvili, and I. Cohen, "Imaging the microscopic structure of shear thinning and thickening colloidal suspensions," Science 333, 1276-1279 (2011).

[22] Mewis, J. and N. J. Wagner, Colloidal Suspension Rheology (Cambridge University Press, Cambridge, 2011).

[23] Boersma, W. H., J. Laven, and H. N. Stein, "Shear thickening (dilatancy) in concentrated dispersions," AIChE J. 36, 321-332 (1990).

[24] Bergenholtz J., J. F. Brady, and M. Vicic, "The non-Newtonian rheology of dilute colloidal suspensions,” J. Fluid Mech. 456, 239-275 (2002).

[25] Fernandez, N., R. Mani, D. Rinaldi, D. Kadau, M. Mosquet, H. Lombois-Burger, J. Cayer-Barrioz, H. J. Herrmann, N. D. Spencer, and L. Isa, "Microscopic mechanism for shear thickening of non-Brownian suspensions," Phys. Rev. Lett. 108301, 1-5 (2013).

[26] Heussinger, C., "Shear thickening in granular suspensions: Interparticle friction and dynamically correlated clusters," Phys. Rev. E 88, 050201 (2013).

[27] Seto, R., R. Mari, J. F. Morris, and M. M. Denn, "Discontinuous shear thickening of frictional hard-sphere suspensions," Phys. Rev. Lett. 111, 218301 (2013).
[28] Brown, M., E. Jaeger, and H. Shear, "Thickening in concentrated suspensions: Phenomenology, mechanisms and relations to jamming," Rep. Prog. Phys. 77, 046602 (2014).

[29] Allen, B., B. Sokol, S. Mukhopadhyay, R. Maharjan, and E. Brown, "System-spanning dynamically jammed region in response to impact of cornstarch and water suspensions," Phys. Rev. E 97, 052603 (2018).

[30] Fall, A., N. Huang, F. Bertrand, G. Ovarlez, and D. Bonn, "Shear thickening of cornstarch suspensions as a reentrant jamming transition," Phys. Rev. Lett. 100, 018301 (2008).

[31] Reynolds, O. "LVII. On the dilatancy of media composed of rigid particles in contact With experimental illustrations," Philos. Mag. Ser. 20, 469-481 (1885).

[32] Metzner, A. B., and M. Whitlock, "Flow behavior of concentrated (dilatant) suspensions," Trans. Soc. Rheol. 2, 239-254 (1958).

[33] Lootens, D., H. van Damme, Y. Hémar, and P. Hébraud, "Dilatant flow of concentrated suspensions of rough particles," Phys. Rev. Lett. 95, 268302 (2005).

[34] Brown, E., and H. M. Jaeger, "Dynamic jamming point for shear thickening suspensions,” Phys. Rev. Lett. 103, 086001 (2009).

[35] Holmes, C. B., M. Fuchs, and M. E. Cates, "Jamming transitions in a schematic model of suspension rheology," Eur. Lett. 63, 240-246 (2003).

[36] Melrose, J. R., and R. C. Ball, “'Contact networks' in continuously shear thickening colloids," J. Rheol. 48, 961-978 (2004).

[37] Wyart, M., and M. E. Cates, "Discontinuous shear thickening without inertia in dense non-Brownian suspensions," Phys. Rev. Lett. 112, 1-5 (2014).

[38] Lin, N. Y. C., B. M. Guy, M. Hermes, C. Ness, J. Sun, W. C. K. Poon, and I. Cohen, "Hydrodynamic and contact contributions to continuous shear thickening in colloidal suspensions," Phys. Rev. Lett. 115, 228304 (2015).

[39] Guy, B. E., M. Hermes, and W. E. E. Poon, "Towards a unified description of the rheology of hard-particle suspensions," Phys. Rev. Lett. 115, 1-5 (2015).

[40] Hsiao, L. C., S. Jamali, D. J. Beltran-Villegas, E. Glynos, P. F. Green, R. G. Larson et al., "A rheological state diagram for rough colloids in shear flow," Phys. Rev. Lett. 119, 158001 (2017).

[41] Jamali, S., A. Boromand, N. Wagner, and J. Maia, "Microstructure and rheology of soft to rigid shear-thickening colloidal suspensions," J. Rheol. 59, 1377-1395 (2015).

[42] Phung, T. N., J. F. Brady, and G. Bossis, "Stokesian dynamics simulation of Brownian suspensions," J. Fluid Mech. 313, 181-207 (1996).

[43] Foss, D. R., and J. F. Brady, "Structure, diffusion and rheology of Brownian suspensions by Stokesian dynamics simulation," J. Fluid Mech. 407, 167 (2000).

[44] Melrose, J. R., and R. C. Ball, "Continuous shear thickening transitions in model concentrated colloids - The role of interparticle forces," J. Rheol. 48, 937 (2004).

[45] Brown, E., and H. M. Jaeger, "The role of dilation and confining stresses in shear thickening of dense suspensions," J. Rheol. 56, 875 (2012).

[46] Byron Bird, R., C. Robert, and O. H. Armstrong, "Dynamics of polymer liquids," in Fluid Mechanics (John Wiley \& Sons, New York, 1987), Vol. 1.

[47] Macosko, C. W., Rheology: Principles, Measurements, and Applications (VCH, New York, 1994).

[48] Cates, M. E., J. P. Wittmer, J.-P. Bouchaud, and C. P. Jamming, "Force chains, and fragile matter," Phys. Rev. Lett. 81, 1841-1844 (1998).

[49] Cates, M. E., M. D. Haw, and C. B. Holmes, "Dilatancy, jamming, and the physics of granulation," J. Phys. Condens. Matter. 17, S2517-S2531 (2005). 
[50] Gurnon, A. K., N. J. Wagner, B. J. Ackerson, J. B. Hayter, N. A. Clark, L. Cotter et al., "Microstructure and rheology relationships for shear thickening colloidal dispersions," J. Fluid Mech. 769, 242-276 (2015).

[51] Cwalina, C. D., and N. J. Wagner, "Material properties of the shearthickened state in concentrated near hard-sphere colloidal dispersions," J. Rheol. 58, 949-967 (2014).

[52] Laun, H. M., "Normal stresses in extremely shear thickening polymer dispersions," J. Nonnewton. Fluid Mech. 54, 87-108 (1994).

[53] Lee, M., M. Alcoutlabi, J. J. Magda, C. Dibble, M. J. Solomon, X. Shi, et al., "The effect of the shear-thickening transition of model colloidal spheres on the sign of $\mathrm{N}_{1}$ and on the radial pressure profile in torsional shear flows," J. Rheol. 50, 293 (2006).

[54] Royer, J. R., D. L. Blair, and S. D. Hudson, "Rheological signature of frictional interactions in shear thickening suspensions," Phys. Rev. Lett. 188301, 1-5 (2016).

[55] Sankaran, A. K., and J. P. Rothstein, "Effect of viscoelasticity on liquid transfer during gravure printing," J Nonnewton. Fluid Mech. 175, 64-75 (2012).

[56] Galindo-Rosales, F. J., M. A. Alves, and M. S. N. Oliveira, "Microdevices for extensional rheometry of low viscosity elastic liquids: A review," Microfluid. Nanofluid. 14, 1-19 (2013).

[57] White E, B., M. Chellamuthu, and J. Rothstein, "Extensional rheology of a shear-thickening cornstarch and water suspension," Rheol. Acta 49, 119-129 (2010).

[58] Chellamuthu, M., E. M. Arndt, and J. P. Rothstein, "Extensional rheology of shear-thickening nanoparticle suspensions," Soft Matter 5, 2117-2224 (2009).

[59] Smith, M. I., R. Besseling, M. E. Cates, and V. Bertola, "Dilatancy in the flow and fracture of stretched colloidal suspensions," Nat. Commun. 1, 114 (2010).

[60] Royall, C. P., W. C. K. Poon, and E. R. Weeks, "In search of colloidal hard spheres," Soft Matter 9, 17-27 (2013).

[61] Maranzano, B. J., and N. J. Wagner, "The effects of particle size on reversible shear thickening of concentrated colloidal dispersions," J. Chem. Phys. 114, 10514-10527 (2001).

[62] Tuladhar, T. R., and M. R. Mackley, "Filament stretching rheometry and break-up behaviour of low viscosity polymer solutions and inkjet fluids,” J. Nonnewton. Fluid Mech. 148, 97-108 (2008).

[63] Rodd, L. E., T. P. Scott, J. J. Cooper-White, and G. H. McKinley, "Capillary break-up rheometry of low-viscosity elastic fluids," Appl. Rheol. 15, 12-27 (2005).

[64] Campo-Deaño, L., and C. Clasen, "The slow retraction method (SRM) for the determination of ultra-short relaxation times in capillary breakup extensional rheometry experiments," J. Nonnewton. Fluid Mech. 165, 1688-1699 (2010).

[65] Galindo-Rosales, F. J., J. P. Segovia-Gutiérrez, F. T. Pinho, M. A. Alves, and J. de Vicente, "Extensional rheometry of magnetic dispersions," J. Rheol. 59, 193-209 (2015).

[66] Niedzwiedz, K., O. Arnolds, N. Willenbacher, and R. Brummer, "How to characterize yield stress fluids with capillary breakup extensional rheometry (CaBER)?," Appl. Rheol. 19, 1-10 (2009).

[67] Spiegelberg, S. H., D. C. Ables, and G. H. McKinley, "The role of end-effects on measurements of extensional viscosity in filament stretching rheometers," J. Nonnewton. Fluid Mech. 64, 229-267 (1996).

[68] McKinley, G. H., and T. Sridhar, "Filament -stretching rheometry of complex fluids," Annu. Rev. Fluid Mech. 34, 375-415 (2002).

[69] Bach, A., H. K. Rasmussen, and O. Hassager, "Extensional viscosity for polymer melts measured in the filament stretching rheometer," J. Rheol. 47, 429-441 (2003).
[70] Marín JM, R., J. K. Huusom, N. J. Alvarez, Q. Huang, H. K. Rasmussen, A. Bach et al., "A control scheme for filament stretching rheometers with application to polymer melts," J. Nonnewton. Fluid Mech. 194, 14-22 (2013).

[71] Orr, N. V., and T. Sridhar, "Probing the dynamics of polymer solutions in extensional flow using step strain rate experiments," J. Nonnewton. Fluid Mech. 82, 203-232 (1999).

[72] Maranzano, B. J., and N. J. Wagner, "The effects of interparticle interactions and particle size on reversible shear thickening: Hard-sphere colloidal dispersions," J. Rheol. 45, 1205-1222 (2001).

[73] Farr, R. S., J. R. Melrose, and R. C. Ball, "Kinetic theory of jamming in hard-sphere startup flows," Phys. Rev. E Stat. Phys. Plasmas Fluids Relat. Interdiscip. Top. 55, 7203-7211 (1997).

[74] Koumakis, N., M. Laurati, S. U. Egelhaaf, J. F. Brady, and G. Petekidis, "Yielding of hard-sphere glasses during start-up shear," Phys. Rev. Lett. 108, 98303 (2012).

[75] Voigtmann, T., "Nonlinear glassy rheology," Curr. Opin. Colloid Interface Sci. 19, 549-560 (2014).

[76] Snijkers, F., and D. Vlassopoulos, "Cone-partitioned-plate geometry for the ARES rheometer with temperature control," J. Rheol. 55, 1167-1186 (2011).

[77] See supplementary material at https://doi.org/10.1122/1.5143653 to observe the results with cone plate and cone-partition-plate (CPP) in steady shear measurements as well as in oscillatory shear. Supplementary material also includes videos sample response during the transient start-up shear experiments of Fig. 7 and extensional experiments depicted in Fig. 9.

[78] Koumakis, N., M. Laurati, A. R. Jacob, K. J. Mutch, A. Abdellali, A. B. Schofield et al., "Start-up shear of concentrated colloidal hard spheres: Stresses, dynamics, and structure," J. Rheol. 60, 603-623 (2016).

[79] Hermes, M., B. M. Guy, and W. C. K. Poon, "Unsteady flow and particle migration in dense, non-Brownian suspensions," J. Rheol. 60, 905 (2016).

[80] Jacob, A. R., E. Moghimi, and G. Petekidis, "Rheological signatures of aging in hard sphere colloidal glasses," Phys. Fluids 31, 087103 (2019).

[81] Mathues, W., C. McIlroy, O. G. Harlen, and C. Clasen, "Capillary breakup of suspensions near pinch-off," Phys. Fluids 27, 093301 (2015).

[82] Sadek, S. H., H. H. Najafabadi, and F. J. Galindo-Rosales, "Capillary breakup extensional magnetorheometry," J. Rheol. 64, 55-65 (2020).

[83] Eggers, J., and E. Villermaux, "Physics of liquid jets," Rep. Prog. Phys. 71, 36601-36679 (2008).

[84] Roché, M., H. Kellay, and H. A. Stone, "Heterogeneity and the role of normal stresses during the extensional thinning of non-Brownian shearthickening fluids,” Phys. Rev. Lett. 107, 134503 (2011).

[85] Furbank, R. J., and J. F. Morris, "An experimental study of particle effects on drop formation,” Phys. Fluids 16, 1777 (2004).

[86] Furbank, R. J., and J. F. Morris, "Pendant drop thread dynamics of particle-laden liquids,” Int. J. Multiph. Flow 33, 448-468 (2007).

[87] Roylance, D., Mechanical Properties of Materials (Massachusetts Institute of Technology, Cambridge, 2008).

[88] Niedzwiedz, K., N. Willenbacher, and R. A. O. Brummer, "How to characterize yield stress fluids with capillary breakup extensional rheometry (CaBER)," Appl. Rheol. 19(4), 41969 (2009).

[89] Herschel, W. H., and R. Bulkley, "Measurement of consistency as applied to rubber-benzene solutions," Proc. Am. Soc. Test Mater. 26, 621 (1926), available at https://www.astm.org/DIGITAL_LIBRARY/ STP/MMR/PAGES/PRO1926-26.htm. 
[90] Nguyen, Q. D. and D. V. Boger, "Measuring the flow properties of yield stress fluids," Annu. Rev. Fluid Mech. 24(1), 47-88 (1992).

[91] Barnes, H. A., "The yield stress-A review or ' $\pi \alpha v \tau \alpha \rho \in \iota^{\prime}-$ Everything flows?, J. Nonnewton. Fluid Mech. 81, 133-178 (1999).

[92] Bonn, D., and M. M. Denn, "Materials science. Yield stress fluids slowly yield to analysis," Science 324, 1401-1402 (2009).

[93] Ovarlez, G., S. Cohen-Addad, K. Krishan, J. Goyon, and P. Coussot, "On the existence of a simple yield stress fluid behavior," J. Nonnewton. Fluid Mech. 193, 68-79 (2013).

[94] Jamming, H. M., "Two-fluid behavior, and 'self-filtration' in concentrated particulate suspensions," Phys. Rev. Lett. 92, 185506 (2004).
[95] Yaoa, M., H. Stephen, and G. H. M. Spiegelbergb, "Dynamics of weakly strain-hardening fluids in filament stretching devices," J. Nonnewton. Fluid Mech. 89, 1-43 (2000).

[96] Rasmussen, H. K., A. G. Bejenariu, O. Hassager, and D. Auhl, "Experimental evaluation of the pure configurational stress assumption in the flow dynamics of entangled polymer melts," J. Rheol. 54, 1325 (2010).

[97] Morrison, F. A., Understanding Rheology (Oxford University, New York, 2001).

[98] Anna, S. L., and G. H. McKinley, "Elasto-capillary thinning and breakup of model elastic liquids," J. Rheol. 45, 115 (2001).

[99] Khandavalli, S., and J. P. Rothstein, "Extensional rheology of shearthickening fumed silica nanoparticles dispersed in an aqueous polyethylene oxide solution,” J. Rheol. 58, 411-431 (2014). 